\title{
Análisis de redes para la transferencia de tecnologías sostenibles entre firmas de construcción internacional
}

\author{
Network Analysis of Green Technology Transfer between International Construction Firms
}

\author{
Rolando-Arturo Cubillos-González \\ Universidad Católica de Colombia. Bogotá (Colombia) \\ Facultad de Diseño, Programa de Arquitectura \\ Centro de investigaciones CIFAR
}

Cubillos-González, R. A. (202). Análisis de redes para la transferencia de tecnologías sostenibles entre firmas de construcción internacional. Revista de Arquitectura (Bogotá), 22(I). 175-186. https://doi. org/10.14718/RevArq.2020.2562

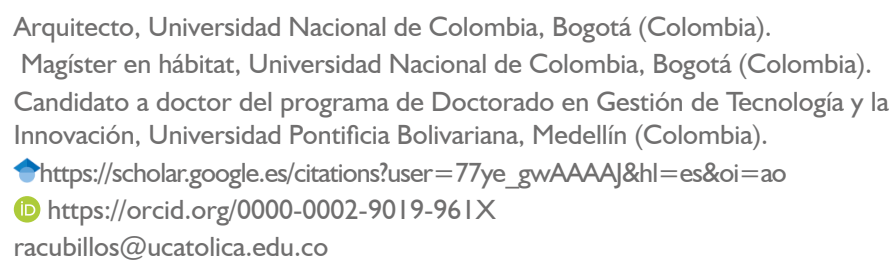

\section{Resumen}

La transferencia de tecnología sostenible es compleja para las firmas de construcción. Una posible solución es analizar esa clase de transferencia como una red social ya que, si se identifican las diferentes relaciones entre los actores del sector construcción, es posible evaluar la capacidad de adaptación tecnológica de dichos actores. El objetivo fue evaluar la transferencia de tecnología sostenible entre empresas constructoras internacionales que se dedican a construir vivienda social o accesible. Para esto, se identificaron dos países con capacidad de transferencia de tecnología sostenible (Reino Unido y Estados Unidos) y dos países de menor capacidad tecnológica y con potencial de adaptarse a dichas tecnologías (Brasil y Colombia); posteriormente, se seleccionaron cinco firmas constructoras por cada país, con las cuales se hizo un análisis de redes (brasilbragrado, intensidad, cercanía y densidad), y luego, procesos de simulación. Como resultado se identificó la capacidad de transferencia tecnológica que tienen las empresas latinoamericanas para aceptar y adaptar tecnologías de empresas de países industrializados, y se espera poder desarrollar indicadores de medición de transferencia tecnológica que permitan comprender mejor la complejidad de la vivienda social.

Palabras clave: adaptación tecnológica; edificaciones sostenibles; industria de la construcción; sector de la construcción; transferencia tecnología; vivienda accesible; vivienda social

\begin{abstract}
The green technology transfer is complex for construction firms. A solution is to analyze it as a social network since, if I identify the different relationships between the actors in the construction sector, it is possible to test the technological adaptation capacity of these actors. The aim was to test the transfer of green technology between international construction companies that dedicated to building social or accessible housing. For this, two countries with the capacity to transfer green technology (United Kingdom and the United States) and two countries with less technological capacity and with the potential to adapt to these technologies (Brazil and Colombia) identified, then 5 construction firms selected for each country with which an analysis of networks (degree, intensity, proximity, and density) and then simulation carried out. As a result, the technological transfer capacity of Latin America companies to accept and adapt technologies from companies in industrialized countries identified, and it hoped to develop indicators of measurement of the technology transfer that allows a better understanding of the complexity of Social Housing.
\end{abstract}

Keywords: technological adaptation; green buildings; construction industry; construction field; technology transfer; affordable housing; social housing.

Recibido: enero 3 / 2019

\section{Introducción}

Este artículo forma parte del proyecto de investigación Tecnologías limpias-fase 2: el impacto de la transferencia de las tecnologías limpias en la vivienda social en Brasil y Colombia, de la línea de investigación en Tecnología, Ambiente y Sostenibilidad, y fue financiado por la Universidad Católica de Colombia.

Para las firmas de construcción, la transferencia de tecnologías sostenibles es un proceso complejo y depende de la relación de distintas variables involucradas. Como ejemplo de lo anterior, la elección de materiales con alto contenido de energía incorporada supone un alto nivel inicial de consumo de energía en la etapa de producción de los edificios, pero también determina el consumo futuro de energía para satisfacer las demandas de calefacción, ventilación y aire acondicionado (Zabalza, Valero \& Aranda, 2011, pp. 1133-1134).

Además, con la perspectiva de las normas de cada país, el consumo de energía en los edificios es un factor clave para alcanzar las metas nacionales e internacionales de emisiones de $\mathrm{CO}_{2}$. En este aspecto, los ahorros económicos para la construcción de edificios son limitados y difíciles de implementar (Zucker et al., 2016, pp. 153-154).

Por otro lado, la renovación de edificios es una oportunidad real para hacer frente a los desafíos actuales de reducción de energía primaria y calentamiento global, pero no es suficiente en términos de tecnología y adaptación sostenible de los 
edificios: así mismo, se identifica que el concepto de equilibrio es central en la implementación de tecnologías orientadas a la eficiencia energética; esto es particularmente cierto en el desarrollo de edificios de cero emisiones de carbono (Sartori, Napolitano \& Voss, 2012, pp. 220-221).

Al respecto, por ejemplo, la energía necesaria para mantener óptimas condiciones ambientales interiores es el $35 \%$ del consumo total de energía en edificios de oficinas (Salcido, Abdul \& Issa, 2016, p. 1008). En contraste, algunos estudios, como el de Pisello et al., (2016, p. 872), evalúan el impacto de la ventilación natural mientras se predice la demanda de energía de los edificios, ya que se afectan sustancialmente la calidad del ambiente interior y el confort térmico.

Algunos autores como Panchal et al. (2016, p. 900) han estudiado la eficiencia energética global de los sistemas de generación única y multigeneracional. Al respecto, se observa que la eficiencia energética del sistema multigeneracional es superior al sistema de generación única. La consecución de los objetivos de sostenibilidad en el sector de la construcción supone adoptar modelos empresariales sostenibles y medidas de eficiencia energética (Moschetti \& Brattebø, 2016, p. 436).

Lo anterior conduce a identificar brechas de investigación en el área. Como respuesta a las crecientes preocupaciones sobre el cambio climático y el medio ambiente, el diseño sostenible de los edificios es cada vez más exigente por parte de los usuarios; sin embargo, la evaluación rápida de varias opciones de diseño requiere la aplicación de herramientas de análisis como modelado de energía, además de las simulaciones de luz y ventilación natural (Niknam \& Karshenas, 2015, p. 910).

Según los autores revisados, el análisis energético exige recopilar información relacionada con la energía de distintas fuentes e introducirla en una aplicación de análisis de energía, lo cual implica un proceso que consume mucho tiempo. Esto causa retrasos y aumenta el tiempo para comparar diferentes alternativas de diseño.

En la actualidad, las decisiones de diseño se reducen, principalmente, a un criterio de eficiencia energética dentro de la evaluación de sostenibilidad de los edificios (Niknam \& Karshenas, 2015, p. 911). Debido a la interdependencia de criterios, el actual enfoque es aplicado de forma lineal, y no tiene en cuenta otros criterios orientados a ver el proceso de diseño de manera holística para un mejor desarrollo de los edificios.
Por lo expuesto, el enfoque sistémico es una mejor alternativa a la evaluación energética de los edificios. Otras propuestas van orientadas a la evaluación económica de los edificios sostenibles y a fomentar su desarrollo (Liu, Guo \& Hu, 2014, p. 37). Tal es el caso de estudios realizados en China que buscan la aplicación de tecnologías de eficiencia energética en los edificios sostenibles.

En ese sentido, la toma de decisiones en proyectos ambientales requiere considerar los impactos sociopolíticos, ambientales y económicos, y ello es a menudo complicado por las diversas opiniones de las partes interesadas (Huang, Keisler \& Linkov, 2011, p. 3579). Según los autores revisados, se hace evidente que el análisis multicriterio es una metodología adecuada para hacer frente a la información técnica disponible y a los valores de las partes interesadas para apoyar las decisiones en muchos campos, y puede ser especialmente valioso en la toma de decisiones ambientales.

Como ejemplo de lo anterior, desde comienzos de la década de 1990 se han propuesto varios sistemas de clasificación de edificaciones sostenibles (Kim, Oh \& Kim, 2013, pp. 203-204). Según los autores, la mayoría de los esfuerzos en esta área se pueden dividir en dos partes principales: 1) desarrollo de sistemas de calificación de edificios sostenibles identificando criterios de calificación pertinentes; 2) evaluación de la validez de los sistemas de calificación de edificios. Los estudios sobre los criterios de calificación de los edificios sostenibles se han centrado, principalmente, en el desarrollo de los ítems de evaluación del rendimiento energético de los edificios.

Otro ejemplo en este tema es la ciudad de Loviisa, en Finlandia, que está planeando una nueva zona residencial sostenible con un total de $240.000 \mathrm{~m}^{2}$ de viviendas residenciales. La ciudad quiere promover soluciones de energía sostenible en la zona considerando varias formas de energía renovable para la calefacción (Kontu et al., 2015, pp. 169-170).

Los resultados de los estudios realizados en tal sentido muestran que la calefacción urbana producida por la cogeneración basada en la biomasa es la alternativa de calentamiento más aceptable, seguida por la bomba de calor de la fuente terrestre, bien sea con información de preferencia o sin ella.

Finalmente, se observa que a partir de esta revisión bibliográfica, es de vital importancia introducir la evaluación de la capacidad de adaptación tecnológica en el área de la construcción con una

This article is available in English on the website of Revista de Arquitectura (Bogotá) https://doi.org/10.14718/RevArq.2020.2562

Network Analysis of Green Technology Transfer between International Construction Firms 
visión de red, ya que eso implica dar un panorama sistematizado del proceso de construcción de una edificación y orientarlo de manera más adecuada hacia la reducción del impacto ambiental y hacia la mejora de su rendimiento energético; sin embargo, este proceso requiere que la aplicación de dicha visión sea aplicativa, para que el sector de la construcción pueda entender e incorporar estos conceptos en sus procesos cotidianos.

Según lo expuesto, por tales motivos incorporar metodologías orientadas a respuestas multiobjetivo puede ser una contribución a los procesos constructivos de una edificación. Por tanto, una posibilidad de comprensión de tal problema es entender este comportamiento complejo como una red social, pues de esa manera es posible estudiar la capacidad de transferencia tecnológica en el campo de la construcción utilizando herramientas para una óptima toma de decisiones.

Según algunos autores del tema, un análisis de redes sociales se puede utilizar como una herramienta de diagnóstico efectiva para hacer explícito un flujo oculto de información valiosa en el sector de la construcción (Alarcón, Alarcón \& Alarcón, 2013, p. 948). Por ejemplo, este tipo de metodología se ha utilizado para examinar cómo el potencial de interferencia existente entre los oficios especializados se relaciona con la confiabilidad del plan de trabajo en las firmas constructoras (Abbasian-Hosseini, Liu \& Hsiang, 2015, p. 145).

Otros estudios se orientaron a analizar, a través de redes, la relación entre el desempeño del proyecto y las características organizacionales en las firmas de construcción (Castillo, Alarcón \& Pellicer, 2018). También, con el análisis de redes se han analizado casos de colaboración entre firmas constructoras coreanas involucradas en proyectos internacionales durante la última década (Park \& Han, 2012, pp. 1460-1461). Igualmente, algunos estudios han utilizado dicha metodología para asimilar la relación entre el Estado y el sector de la construcción en la aplicación de las políticas públicas en Brasil (Marques, Bissoli-Dalvi \& Alvarez, 2018, p. 187); sin embargo, de acuerdo con la revisión literaria, no se observan estudios relacionados con el análisis de redes y el estudio de la trasferencia de tecnología aplicado a las firmas constructoras, lo cual indica un vacío de conocimiento que debe ser estudiado.

El objetivo de este artículo fue evaluar la transferencia de tecnologías sostenibles entre empresas constructoras internacionales que se dedican a construir vivienda social, para el caso latinoamericano, o vivienda accesible, para el caso europeo y de Estados Unidos. En el presente estudio se utilizaron como metodología el análisis de redes y la simulación.

El resultado fue una simulación a partir del análisis de redes de la capacidad de las firmas constructoras seleccionadas internacionalmente, para hacer diferentes transferencias de tecnologías sos- tenibles entre ellas. Con este ejercicio se espera desarrollar a futuro indicadores de medición del proceso de transferencia tecnológica en el campo de la construcción que permitan comprender mejor la complejidad de este proceso en el área de la vivienda social. La estructura del artículo es la siguiente: primero se explican la metodología desarrollada y las actividades Ilevadas a cabo para lograr el objetivo propuesto. En segundo lugar, se describen los resultados del estudio. Finalmente, se exponen las conclusiones del artículo.

\section{Metodología}

El presente estudio busca confirmar la hipótesis de que si se identifican las diferentes relaciones entre los actores del sector construcción que tienen capacidad de transferencia tecnológica para el desarrollo de vivienda social o accesible a escala internacional, entonces es posible evaluar la capacidad de adaptación tecnológica de dichos actores.

Para el diseño de la red se definió un tipo de red de intercambio, con el objeto de analizar la capacidad de transferencia de tecnología de las diferentes firmas constructoras. En un nivel macro se identificaron las relaciones de la estructura de la red. En un nivel micro se identificaron los cambios que tiene una tecnología al ser transferida por parte de una firma constructora. El estudio fue conformado por tres fases de investigación organizadas como se muestra en la figura 1.

- Obtención de datos: en esta fase se realizaron las siguientes actividades: en primer lugar, se identificaron dos países de mayor capacidad tecnológica; en este caso, Reino Unido y Estados Unidos. Luego, se identificaron dos países de menor capacidad tecnológica; en este caso, Brasil y Colombia (McKinsey Global Institute, 2017, p. 13). A continuación, se seleccionaron 20 firmas constructoras; 5, por cada país (figura 2). Para la selección de cada firma constructora se identificó su capacidad tecnológica, al igual que si dichas firmas realizaban proyectos de vivienda social y vivienda en general. Así mismo, las firmas constructoras fueron identificadas a partir de los rankings de las mejores firmas constructoras realizados por las entidades gubernamentales de los países objeto de estudio.

- Simulación de datos: para la simulación de datos se definieron atributos y variables. Luego, se construyó una matriz de datos, denominada RED-0, que representa un tiempo inicial T0. A continuación, se hicieron tres simulaciones matriciales, denominadas RED-1, RED-2 y RED-3; cada una, con tiempos T1, T2 y T3. Finalmente, se graficaron las cuatro redes.

- Análisis de redes: para el análisis de redes se calcularon el grado, la intensidad y la cercanía de red, y luego, su densidad general. Después se identificaron las firmas constructoras más influyentes y las menos influyentes 


\section{Fases metodológicas}

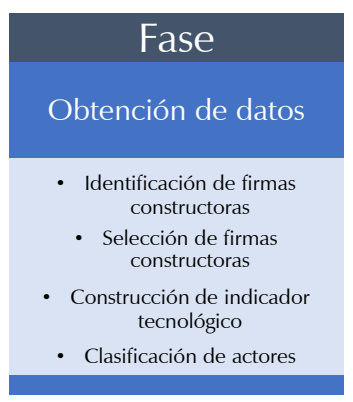

1

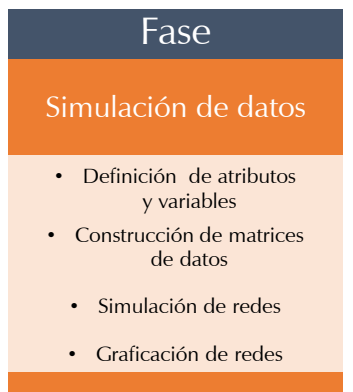

2

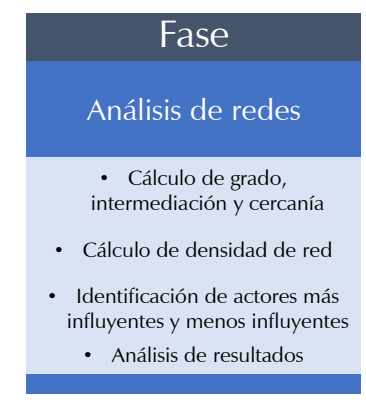

3

(A) Figura 1. Fases

metodológicas del estudio.

Fuente: elaboración propia (2019).

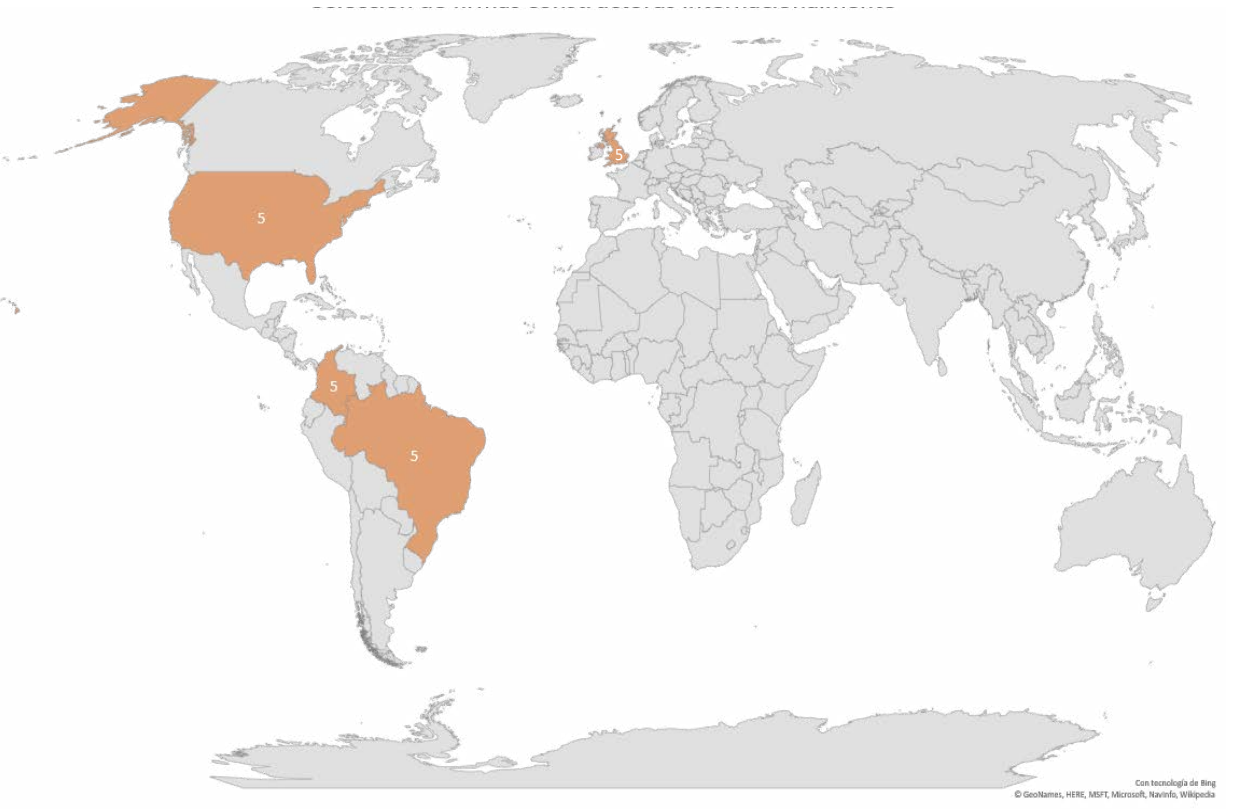

(A) Figura 2. Selección de firmas constructoras Internacionalmente

Fuente: elaboración propia (2019) de cada simulación. Con estas mediciones se pudo identificar y determinar la capacidad de transferencia de tecnologías sostenibles de las firmas constructoras de países con alta capacidad tecnológica y las firmas constructoras de menor capacidad en esta área. Finalmente, se hizo un análisis de resultados.

El presente estudio centró su análisis en la construcción de relaciones y características entre el factor dependiente, denominado actores con capacidad de transferencia tecnológica, y los denominados factores independientes. Estos son: el impacto ambiental, el rendimiento energético de la edificación y el Código de Identificación Tecnológica. Para ello se diseñó un indicador que permitió establecer la relación y el efecto de cada uno de los factores que intervienen en la transferencia tecnológica de una firma constructora. Cada una de las variables se definió de la siguiente manera:

\section{- Actores con capacidad de transferencia tecno-} lógica: son los actores con capacidad de acciones de transferencia de una tecnología con el fin de que esta sea más eficiente en las condiciones de un contexto y un ambiente determinados. En el caso particular de las firmas constructoras, esta variable se refiere a las tecnologías que se orientan a las energías renovables por sobre las convencionales. Por ejemplo, algunas tecnologías de ahorro de energía pasivas y activas de bajo costo se han utilizado con éxito por parte de firmas constructoras en una transformación tecnológica de ahorro de energía en la producción de oficinas en Tianjin. Los resultados muestran que el consumo de energía y el mantenimiento representan la mayor parte del costo del ciclo de vida (Ma et al., 2016, pp. 810-811). Otro ejemplo es la flexibilidad del sistema eléctrico aprovechando las capacidades de generación de energía renovable en los edificios, lo que es de gran importancia para un desarrollo sostenible. Los sistemas de control para implementar dichas medidas de respuesta a la demanda necesitan cuantificar la flexibilidad de los respectivos edificios (Lopes et al., 2016, p. 1053).

- Impacto ambiental: es el efecto causado por las actividades del hombre sobre el ambiente. En el caso particular de las firmas constructoras, esta definición se refiere al consumo energético de los materiales y los procesos constructivos. Al respecto, la revisión bibliográfica encontró que, por ejemplo, el Código Nacional de Construcción de Holanda propone un método fácilmente aplicable que permite un diseño de edificios optimizado respecto a los impactos ambientales (Alsema et al., 2016, pp. 519-522). El enfoque propuesto es una evaluación del rendimiento integral de la construcción respecto a la demanda de energía y materiales durante el ciclo de vida del edificio. Por tanto, extender este enfoque para la evaluación de los procesos de construcción a otros países parece un paso lógico, ya que ofrece a los diseñadores una mejor visión del rendimiento total del edificio. Por otro lado, la adaptación de los edificios existentes ofrece importantes oportunidades para mejorar la comodidad y el bienestar de los ocupantes, y ello se está considerando uno de los principales enfoques para lograr la sostenibilidad en el entorno construido a un costo relativamente bajo y con altas tasas de absorción (Asadi et al., 2014, pp. 444-445). En este sentido, los autores revisados proponen un modelo de optimización multiobjetivo que utiliza un algoritmo genético y artificial, redes neuronales para evaluar cuantitativamente las opciones tecnológicas en un proyecto de rehabilitación de edificios.

- Rendimiento energético de la edificación: es la reducción del consumo de energías convencionales en una edificación con el fin de ahorrar y hacer un uso racional de dichas energías, además, del consumo de energía en los procesos de construcción y operación. Por ejemplo, la simulación del desempeño del edificio se utiliza frecuentemente para apoyar el diseño, la renovación y el funcionamiento del edificio (Carlucci et al., 2016, pp. 280-281); sin embargo, tradicionalmente los modeladores se ocupan de describir con precisión los datos técnicos de entrada, y solo tienen un interés limitado en investigar la influencia del comportamiento de 
los ocupantes en el rendimiento energético de los edificios. Otros autores hacen énfasis en el rendimiento térmico, energético y de confort de las fachadas de doble piel, que se desempeñan mejor en comparación con las fachadas de doble o de triple acristalamiento (Gelesz \& Reith, 2015, pp. 558-559).

A partir de las anteriores definiciones, se estableció el siguiente indicador, para poder evaluar la capacidad de adaptación tecnológica en las edificaciones:

Indicador de actores con capacidad de transferencia tecnológica (IACTT) = impacto ambiental tecnológico (IAT) + rendimiento energético de la edificación (REE) + Código de Identificación Tecnológica (CIT)

$$
(\mathrm{IACTT})=(\mathrm{IAT})+(\mathrm{REE})+(\mathrm{CIT})
$$

Así mismo, para hacer el análisis de red del proceso de transferencia tecnológica de un actor que representa una firma constructora se establecieron los siguientes nodos:

- Actor con capacidad de transferencia de tecnologías tradicionales: es el individuo que representa a una firma de construcción que utiliza tecnologías tradicionales de alto impacto ambiental.

- Actor con capacidad de transferencia de tecnologías de aislamiento térmico: es el individuo que representa a una firma de construcción que utiliza tecnologías a partir de estrategias pasivas.
- Actor con capacidad de transferencia de tecnologías de eficiencia energética: es el individuo que representa a una firma de construcción que utiliza tecnologías que reducen el consumo energético y las combina con estrategias pasivas.

- Actor con capacidad de transferencia de tecnologías limpias: es el individuo que representa a una firma de construcción que utiliza tecnologías que reducen los impactos ambientales a través del análisis de todo el ciclo de vida, tanto de los recursos como en los procesos utilizados en el diseño, la producción y la operación de una edificación.

- Actor con capacidad de transferencia de tecnologías sensibles al clima: es el individuo que representa a una firma de construcción que utiliza tecnologías computacionales para identificar patrones de comportamiento del cambio climático que pueden ser utilizados en los procesos de diseño, construcción y operación de una edificación.

- Actor con capacidad de transferencia de tecnologías bajas en carbono: es el individuo que representa a una firma de construcción que utiliza tecnologías que producen un mínimo de gases de efecto invernadero a lo largo del diseño, la construcción y la operación de una edificación.

Por lo anterior, para el desarrollo del experimento, se estableció que el universo de la red inicial fuera de 20 nodos, los cuales representaban a las 20 firmas constructoras seleccionadas previamente (tabla 1). Luego, la organización y la

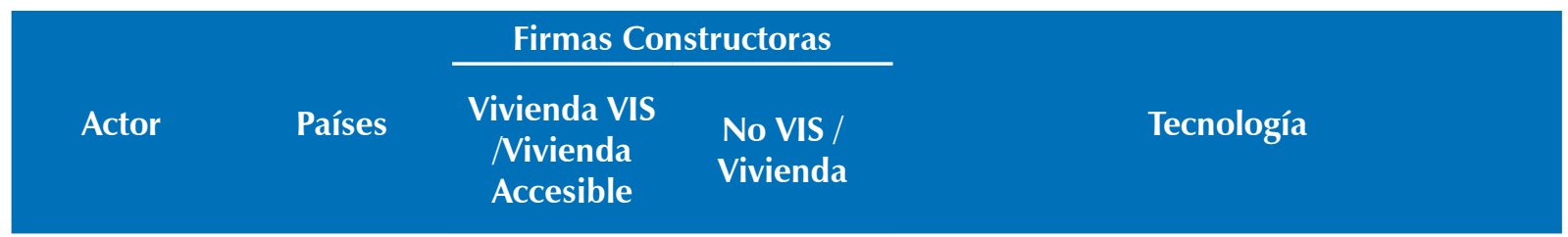

\begin{tabular}{|c|c|c|c|c|}
\hline A1 & UK & $X$ & $X$ & Tecnologías sensibles al clima \\
\hline A2 & $\mathrm{COL}$ & $X$ & $X$ & Tecnologías tradicionales \\
\hline A3 & $\mathrm{COL}$ & $X$ & $X$ & Tecnologías de aislamiento térmico \\
\hline A4 & $\mathrm{COL}$ & $X$ & $X$ & Tecnologías tradicionales \\
\hline A5 & UK & $X$ & $X$ & Tecnologías sensibles al clima \\
\hline A6 & UK & $X$ & $X$ & Tecnologías bajas en carbono \\
\hline A7 & UK & $X$ & $X$ & Tecnologías de eficiencia energética \\
\hline A8 & BRA & $X$ & $X$ & Tecnologías tradicionales \\
\hline A9 & UK & $X$ & $X$ & Tecnologías de eficiencia energética \\
\hline A10 & $\mathrm{BRA}$ & $X$ & $X$ & Tecnologías tradicionales \\
\hline A 11 & USA & $X$ & $X$ & Tecnologías de eficiencia energética \\
\hline A 12 & $\mathrm{COL}$ & $X$ & $X$ & Tecnologías de aislamiento térmico \\
\hline A13 & $\mathrm{BRA}$ & $X$ & $x$ & Tecnologías de aislamiento térmico \\
\hline A14 & BRA & $x$ & $X$ & Tecnologías de aislamiento térmico \\
\hline A15 & USA & $x$ & $X$ & Tecnologías sensibles al clima \\
\hline A16 & USA & $x$ & $X$ & Tecnologías de aislamiento térmico \\
\hline A17 & $\mathrm{COL}$ & $x$ & $x$ & Tecnologías tradicionales \\
\hline A18 & $\mathrm{BRA}$ & $x$ & $x$ & Tecnologías tradicionales \\
\hline A19 & USA & $x$ & $X$ & Tecnologías sensibles al clima \\
\hline A20 & USA & $X$ & $X$ & Tecnologías bajas en carbono \\
\hline
\end{tabular}

Tabla 1. Firmas constructoras seleccionadas e identificación del uso de las tecnologías sostenibles. Fuente: elaboración propia (2019). 
(17) Tabla 2. Parametrización de (1) variables para construcción de indicador.

Fuente: elaboración propia (2019).

(7) Tabla 3. Parametrización de (v) variables para construcción de indicador.

Fuente: elaboración propia (2019). clasificación de las firmas constructoras se hicieron de manera aleatoria. Para validar la hipótesis propuesta a partir del indicador diseñado, se recurrió a una calificación de las tecnologías usadas por cada una de las firmas constructoras seleccionadas de acuerdo con la información suministrada por las bases de datos y las páginas web de cada firma. Los nodos de análisis fueron numerados de 1 a 6 , para que se pudiese identificarlos. Así mismo, se estableció una parametrización de las variables a través de una puntuación de 1 a 60, organizada en 6 deciles para las variables independientes (tabla 2). Cabe anotar que la métrica diseñada en ese estado inicial del trabajo solo tuvo en cuenta números enteros positivos.

Por otra parte, a la relación entre variables siguió un proceso de coherencia en el proceso de calificación. Por ejemplo, a mayor impacto ambiental (calificación 6), menor eficiencia energética (calificación 1); es decir, la relación entre estas dos variables es inversamente proporcional. Como resultado se obtuvo una calificación de cada actor entre 8 y 13 (tabla 3), donde 8 era el puntaje del menor indicador relacionado con la tecnología de mayor impacto y menor eficiencia, en comparación con 13, que era el puntaje del mayor indicador relacionado con la tecnología de menor impacto y mayor eficiencia.

Seguidamente, se procedió a la simulación. Para ello se realizaron cuatro matrices de doble entrada y se corrió el modelo, con el cual se generaron cuatro escenarios de red, y que permitió identificar los distintos agentes y sus relaciones. En tal sentido, la identificación de cada nodo se hizo a partir de establecer que 0 equivale a ninguna relación, y 1 , al número de entradas que tiene un nodo específico dentro del universo propuesto.

\section{Resultados}

Finalmente, los resultados del experimento fueron validados en el programa UCINET 6.682, en el cual se graficaron las diferentes redes y se estudió la cohesión de los factores de impacto ambiental y rendimiento energético frente a la transferencia tecnológica, a través del análisis del grado (degree), la intermediación (betweenness) y la cercanía (closeness). Estas tres unidades de medida permitieron identificar y medir el nivel de la capacidad que se presenta en el proceso de transferencia tecnológica de una firma constructora (Borgatti, Everett \& Johnson, 2013).

Así mismo, se hizo el análisis de la densidad, el cual identificó el número de relaciones posibles entre nodos, lo que, a su vez, visualizó el comportamiento general que se presenta al transferir una tecnología de una firma constructora a otra. Mientras, el estudio del grado mostró el número de relaciones entre las variables independientes, que permiten medir el comportamiento individual de cada factor frente al proceso de transferencia tecnológica.

\begin{tabular}{|c|c|c|c|c|c|}
\hline $\begin{array}{c}\text { Impacto } \\
\text { ambiental } \\
\text { tecnológico } \\
\text { (IAT) }\end{array}$ & Valor & $\begin{array}{l}\text { Rendimiento } \\
\text { de la } \\
\text { edificación } \\
\text { (REE) }\end{array}$ & Valor & Actores con capacidad de transferencia tecnológica (ATT) & $\begin{array}{c}\text { Código de } \\
\text { Identificación } \\
\text { Tecnológica (CIT) }\end{array}$ \\
\hline $0-10$ & 1 & $0-10$ & 1 & Actor con capacidad de transferencia de tecnologías tradicionales & 1 \\
\hline $11-20$ & 2 & $11-20$ & 2 & Actor con capacidad de transferencia de tecnologías de aislamiento térmico & 2 \\
\hline $21-30$ & 3 & $21-30$ & 3 & Actor con capacidad de transferencia de tecnologías de eficiencia energética & 3 \\
\hline $31-40$ & 4 & $31-40$ & 4 & Actor con capacidad de transferencia de tecnologías limpias & 4 \\
\hline $41-50$ & 5 & $41-50$ & 5 & Actor con capacidad de transferencia de tecnologías sensibles al clima & 5 \\
\hline $51-60$ & 6 & $51-60$ & 6 & Actor con capacidad de transferencia de tecnologías bajas en carbono & 6 \\
\hline
\end{tabular}

\begin{tabular}{|c|c|c|c|c|}
\hline ACTT & $\begin{array}{c}\text { Impacto } \\
\text { ambiental } \\
\text { tecnológico (IAT) }\end{array}$ & $\begin{array}{l}\text { Rendimiento } \\
\text { energético de la } \\
\text { edificación (REE) }\end{array}$ & $\begin{array}{c}\text { Código de } \\
\text { identificación } \\
\text { tecnológica }(\mathrm{CIT})\end{array}$ & $\begin{array}{l}\text { Indicador de actores con } \\
\text { capacidad de transferencia } \\
\text { tecnológica (IACTT) }\end{array}$ \\
\hline
\end{tabular}

Actor con capacidad de transferencia de tecnologías tradicionales

Actor con capacidad de transferencia de tecnologías basadas en estrategias pasivas Actor con capacidad de transferencia de tecnologías de eficiencia energética Actor con capacidad de transferencia de tecnologías limpias

Actor con capacidad de transferencia de tecnologías sensibles al clima

Actor con capacidad de transferencia de tecnologías bajas en carbono

\begin{tabular}{|c|c|c|c|}
\hline 6 & 1 & 1 & 8 \\
\hline 5 & 2 & 2 & 9 \\
\hline 4 & 3 & 3 & 10 \\
\hline 3 & 4 & 4 & 11 \\
\hline 2 & 5 & 5 & 12 \\
\hline 1 & 6 & 6 & 13 \\
\hline
\end{tabular}




\begin{tabular}{|c|c|c|c|c|c|c|c|c|c|}
\hline Actor & País & IAT & REE & CIT & IACTT & Grado & Intermediación & Cercanía & $\begin{array}{c}\text { Capacidad transferencia } \\
\text { tecnológica } \\
\text { CTT = } \\
\left.\text { (grado n. } .^{\circ} / \text { actores }\right)\end{array}$ \\
\hline $\mathrm{A} 1$ & UK & 3 & 4 & 4 & 11 & 13 & 1,52 & 25 & $65 \%$ \\
\hline A2 & $\mathrm{COL}$ & 6 & 1 & 1 & 8 & 15 & 2,595 & 23 & $75 \%$ \\
\hline A3 & $\mathrm{COL}$ & 5 & 2 & 2 & 9 & 16 & 3,085 & 22 & $80 \%$ \\
\hline A4 & $\mathrm{COL}$ & 6 & 1 & 1 & 8 & 17 & 2,932 & 21 & $85 \%$ \\
\hline A5 & UK & 2 & 5 & 5 & 12 & 13 & 1,304 & 25 & $65 \%$ \\
\hline A6 & UK & 1 & 6 & 6 & 13 & 15 & 2,113 & 23 & $75 \%$ \\
\hline A7 & UK & 4 & 3 & 3 & 10 & 14 & 1,831 & 24 & $70 \%$ \\
\hline A8 & BRA & 6 & 1 & 1 & 8 & 15 & 2,947 & 23 & $75 \%$ \\
\hline A9 & UK & 4 & 3 & 3 & 10 & 13 & 1,266 & 25 & $65 \%$ \\
\hline $\mathrm{A} 10$ & BRA & 6 & 1 & 1 & 8 & 19 & 4,181 & 19 & $95 \%$ \\
\hline A11 & USA & 4 & 3 & 3 & 10 & 14 & 1,936 & 24 & $70 \%$ \\
\hline $\mathrm{A} 12$ & $\mathrm{COL}$ & 5 & 2 & 2 & 9 & 15 & 2,249 & 23 & $75 \%$ \\
\hline A13 & BRA & 5 & 2 & 2 & 9 & 12 & 1,357 & 26 & $60 \%$ \\
\hline A14 & BRA & 5 & 2 & 2 & 9 & 18 & 3,403 & 20 & $90 \%$ \\
\hline A15 & USA & 2 & 5 & 5 & 12 & 13 & 1,314 & 25 & $65 \%$ \\
\hline A16 & USA & 5 & 2 & 2 & 9 & 13 & 1,314 & 25 & $65 \%$ \\
\hline A17 & $\mathrm{COL}$ & 6 & 1 & 1 & 8 & 14 & 1,602 & 24 & $70 \%$ \\
\hline A18 & BRA & 6 & 1 & 1 & 8 & 14 & 1,585 & 24 & $70 \%$ \\
\hline A19 & USA & 3 & 4 & 4 & 11 & 16 & 2,41 & 22 & $80 \%$ \\
\hline A20 & USA & 1 & 6 & 6 & 13 & 15 & 2,056 & 23 & $75 \%$ \\
\hline
\end{tabular}

Tabla 4. Resultados, RED-0. Fuente: elaboración propia (2019).

\section{Resultados de La RED-0}

La red inicia con un tiempo To y con 20 actores, de los cuales se observa que las 5 principales firmas de la red son todas latinoamericanas e inician con una alta capacidad de transferencia tecnológica. En este caso, una alta capacidad para recibir tecnologías sostenibles. Se identifica que la firma constructora A10 representa a una compañía de Brasil con una alta capacidad de transferencia tecnológica del $95 \%$ y un IACTT de 8; es decir, posee tecnologías tradicionales. En este caso en particular, se observa que la firma tiene una alta capacidad de relación con otros actores de la red, o sea que su grado es de 19 y tiene una alta capacidad de transmisión y recepción de tecnología. Su intermediación es de 4,181. Así mismo, su cercanía es de 19, lo cual le permite interactuar con otros actores fácilmente, ya que se encuentra ubicada en el centro de la red. Por otro lado, en la tabla 4 se pueden observar los 5 primeros actores de la RED-0 y los resultados correspondientes. La figura 3 muestra la graficación de la RED-0 y la figura 4 presenta el comportamiento gráfico de los datos de la tabla 4.

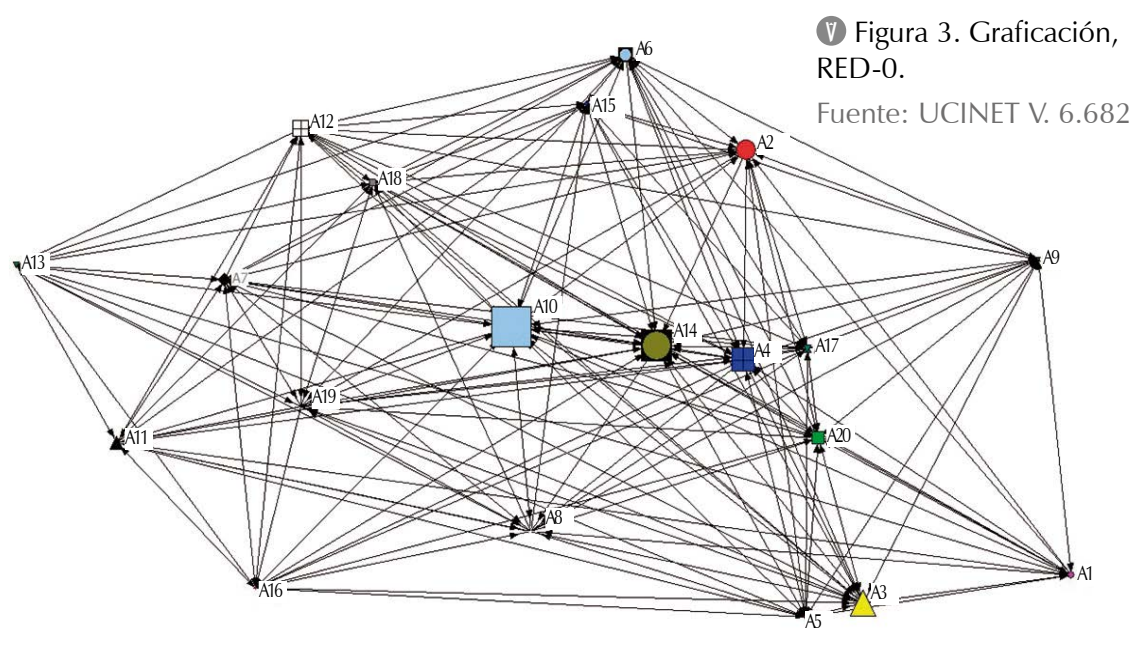

Actores con Capacidad de Transferencia Tecnológica (ACTT) RED 0 - TO

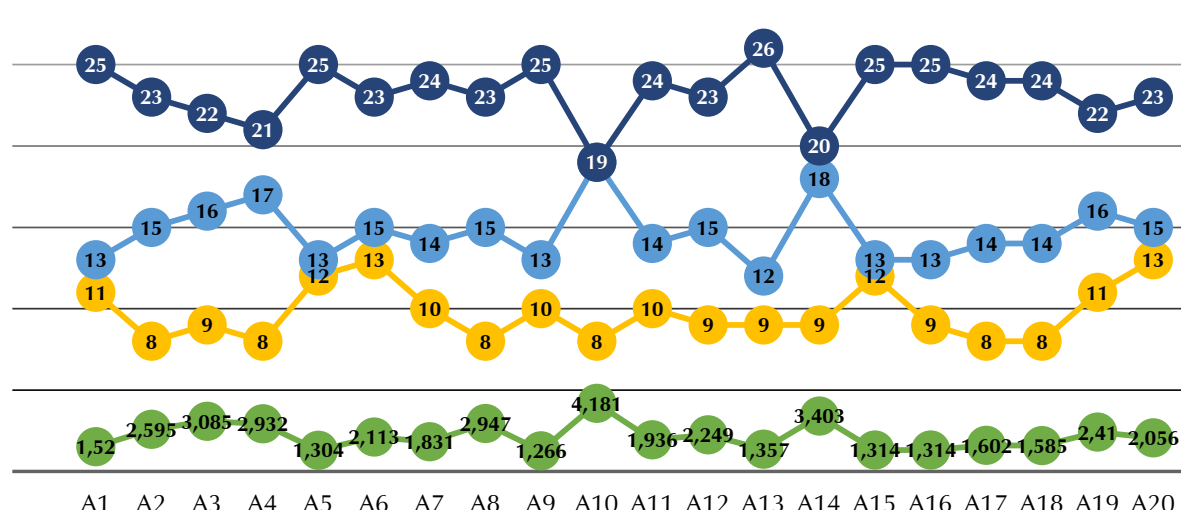

$\rightarrow$ IACTT $\rightarrow$ Grado $\rightarrow$ Intermediación $\rightarrow$ Cercanía 
Capacidad de transferencia Actor País IAT REE CIT IACTT Grado Intermediación Cercanía tecnológica CTT = (Grado/

\begin{tabular}{|c|c|c|c|c|c|c|c|c|c|}
\hline & & & & & & & & & - actores) \\
\hline A1 & UK & 1 & 6 & 6 & 13 & 12 & 8,776 & 18 & $75 \%$ \\
\hline A2 & $\mathrm{COL}$ & 4 & 3 & 3 & 10 & 8 & 4,093 & 22 & $63 \%$ \\
\hline A3 & $\mathrm{COL}$ & 2 & 5 & 5 & 12 & 12 & 13,85 & 18 & $75 \%$ \\
\hline A4 & $\mathrm{COL}$ & 3 & 4 & 4 & 11 & 10 & 5,367 & 20 & $63 \%$ \\
\hline A6 & UK & 2 & 5 & 5 & 12 & 7 & 1,843 & 23 & $44 \%$ \\
\hline A7 & UK & 4 & 3 & 3 & 10 & 7 & 2,283 & 23 & $44 \%$ \\
\hline A8 & BRA & 3 & 4 & 4 & 11 & 10 & 7,793 & 20 & $63 \%$ \\
\hline A10 & BRA & 2 & 5 & 5 & 12 & 11 & 6,817 & 19 & $69 \%$ \\
\hline A11 & USA & 2 & 5 & 5 & 12 & 4 & 0,167 & 26 & $25 \%$ \\
\hline A12 & $\mathrm{COL}$ & 3 & 4 & 4 & 11 & 6 & 0,676 & 24 & $38 \%$ \\
\hline A13 & BRA & 4 & 3 & 3 & 10 & 3 & 0,167 & 27 & $19 \%$ \\
\hline A14 & BRA & 3 & 4 & 4 & 11 & 6 & 0,476 & 24 & $38 \%$ \\
\hline A17 & COL & 4 & 3 & 3 & 10 & 4 & 0,167 & 26 & $25 \%$ \\
\hline A18 & BRA & 3 & 4 & 4 & 11 & 5 & 0,367 & 25 & $31 \%$ \\
\hline A19 & USA & 4 & 3 & 3 & 10 & 7 & 1,66 & 23 & $44 \%$ \\
\hline A20 & USA & 2 & 5 & 5 & 12 & 8 & 5,5 & 22 & $50 \%$ \\
\hline
\end{tabular}

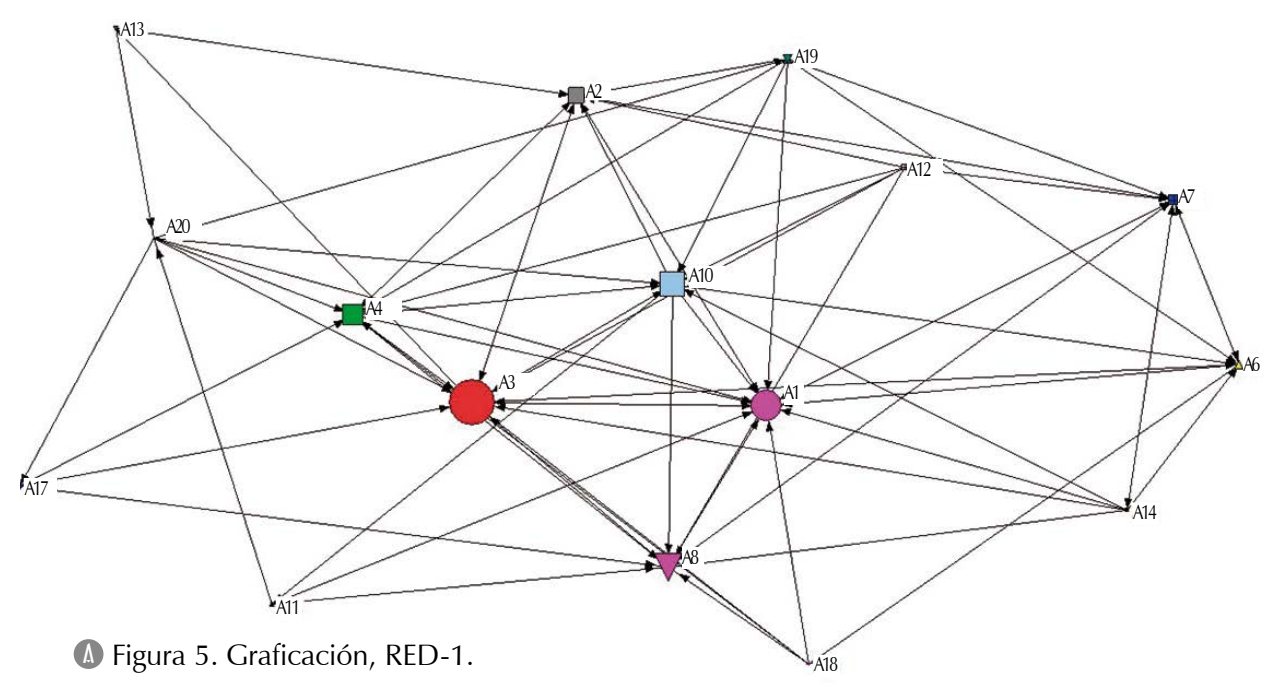

Fuente: UCINET V. 6.682

Actores con Capacidad de Transferencia Tecnológica (ACTT) RED 1 - T1
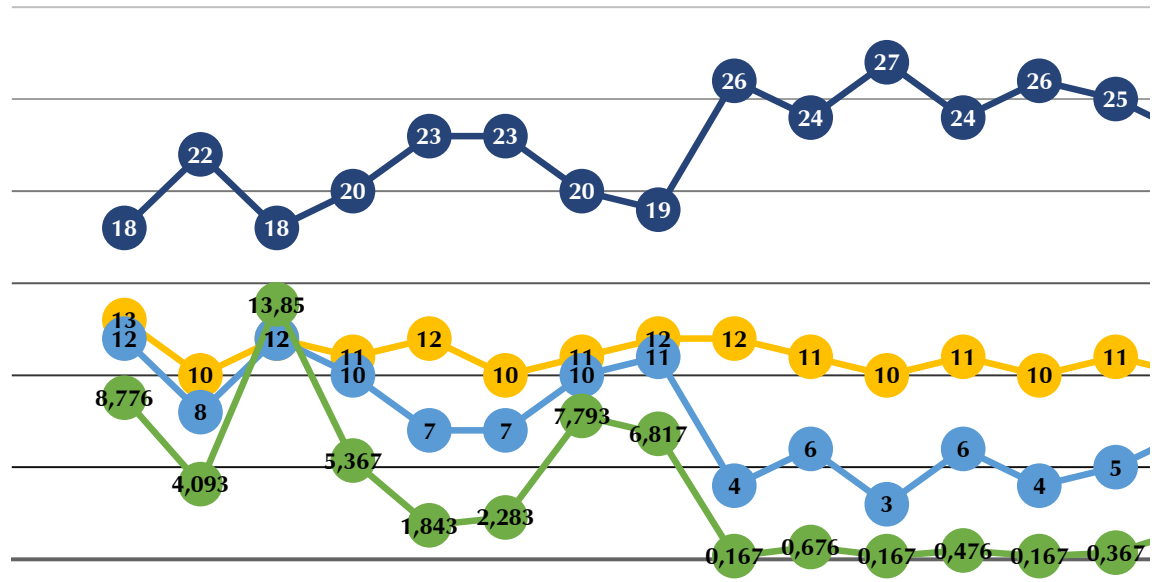

$\begin{array}{llllllllllllll}\text { A1 } & \text { A2 } & \text { A3 } & \text { A4 } & \text { A6 } & \text { A7 } & \text { A8 } & \text { A10 } & \text { A11 } & \text { A12 } & \text { A13 } & \text { A14 } & \text { A17 } & \text { A18 }\end{array}$

$\multimap$ IACTT $\rightarrow$ Grado $\multimap$ Intermediación $\multimap$ Cercanía

(A) Figura 6. Graficación, RED-0.

Fuente: UCINET V. 6.682

\section{Resultados de La RED-1}

Después de eliminar las firmas constructoras que tienen las menores capacidades de transferencia tecnológica, la red inicia con un tiempo T1 y con 16 actores. Se observa que las 5 firmas principales firmas de la red son: una europea y cuatro latinoamericanas, que comienzan con una alta capacidad de transferencia tecnológica. Así mismo, se identifica que, a partir de la simulación realizada, las firmas constructoras han evolucionado en el uso del tipo de tecnología. También se observa la presencia de una firma constructora con una alta capacidad para transferir tecnologías, y la de 4 firmas constructoras con una alta capacidad para recibir dichas tecnologías. En este caso, la firma constructora A1 representa a una compañía del Reino Unido con una capacidad de transferencia tecnológica del $75 \%$ y un IACTT de 13; es decir, posee tecnologías bajas en carbono. Además, dicha firma está a la par con una firma constructora A3 que representa a una compañía de Colombia con una capacidad de transferencia tecnológica del $75 \%$ y un IACTT de 12, según lo cual posee una tecnología sensible al clima. Es importante anotar que dicha firma ha evolucionado en el proceso de simulación de un IACTT de 9 (tabla 4) a un IACTT de 12 (tabla 5), lo cual le permite tener la capacidad para aceptar nuevas tecnologías. En la tabla 5 se pueden observar los 5 primeros actores de la RED-1 y los resultados correspondientes. La figura 5, por su parte, muestra la graficación de la RED-1, y la figura 6 presenta el comportamiento grafico de los datos de la tabla 5. 


\begin{tabular}{|c|c|c|c|c|c|c|c|c|c|}
\hline Actor & País & IAT & REE & CIT & IACTT & Grado & Intermediación & Cercanía & $\begin{array}{c}\text { Capacidad de transferencia } \\
\text { tecnológica CTT }=(\text { Grado/ } \\
\left.\text { n. }{ }^{\circ} \text { actores }\right)\end{array}$ \\
\hline A1 & UK & 1 & 6 & 6 & 13 & 3 & 0,667 & 22 & $25 \%$ \\
\hline $\mathrm{A} 2$ & $\mathrm{COL}$ & 1 & 6 & 6 & 13 & 5 & 0,583 & 19 & $42 \%$ \\
\hline A3 & $\mathrm{COL}$ & 3 & 4 & 4 & 11 & 7 & 11,5 & 15 & $58 \%$ \\
\hline A4 & $\mathrm{COL}$ & 2 & 5 & 5 & 12 & 4 & 1,917 & 19 & $33 \%$ \\
\hline A6 & UK & 4 & 3 & 3 & 10 & 6 & 4,917 & 16 & $50 \%$ \\
\hline A7 & UK & 3 & 4 & 4 & 11 & 3 & 2,333 & 21 & $25 \%$ \\
\hline A8 & BRA & 2 & 5 & 5 & 12 & 2 & 0,000 & 23 & $17 \%$ \\
\hline A10 & BRA & 4 & 3 & 3 & 11 & 7 & 6,417 & 15 & $58 \%$ \\
\hline A12 & $\mathrm{COL}$ & 4 & 3 & 3 & 10 & 4 & 2,167 & 19 & $33 \%$ \\
\hline A14 & BRA & 2 & 5 & 5 & 12 & 7 & 4,833 & 15 & $58 \%$ \\
\hline A19 & USA & 4 & 3 & 3 & 10 & 7 & 6,583 & 15 & $58 \%$ \\
\hline A20 & USA & 1 & 6 & 6 & 13 & 3 & 2,083 & 21 & $25 \%$ \\
\hline
\end{tabular}

\section{Resultados de La RED-2}

Después de una segunda eliminación de las firmas constructoras que tienen las menores capacidades de transferencia tecnológica, la red inicia con un tiempo T2 y con 12 actores. Se observa que las 5 principales firmas de la red son: una europea, una de Estados Unidos y 3 latinoamericanas. Se observa que las 5 poseen capacidades similares de transferencia tecnológica. Así mismo, se identifica que, a partir de la simulación realizada, las firmas constructoras han continuado su evolución en el uso de diferentes tipos de tecnología. En esta red se observa que la firma constructora con una alta capacidad para transferir tecnologías es la firma colombiana A3, que tiene una capacidad de transferencia tecnológica del $58 \%$ y un IACTT de 11. Es importante anotar que en este momento la firma retrocede una tecnología: pasa de un IACTT de 12 a un IACTT de 11; es decir, la firma perdió capacidad de transferencia en el proceso. Sin embargo, la firma tiene una alta intermediación de 11,5, o sea que la empresa tiene una alta capacidad de transmisión y recepción tecnológica dentro de la red, lo cual la hace un buen intermediador frente a otros actores en la red (figura 7). En la tabla 6 se pueden observar los 5 primeros actores de la RED-2 y los resultados correspondientes. La figura 7 muestra la graficación de la RED-1 y la figura 8 presenta el comportamiento grafico de los datos de la tabla 6 .

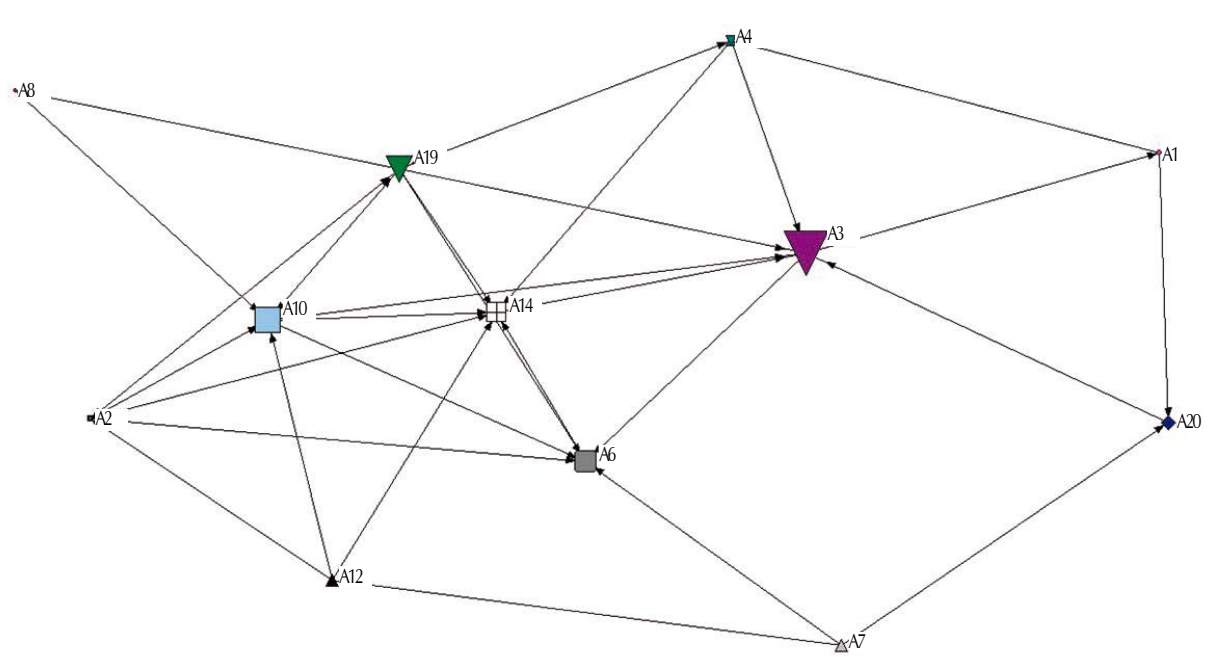

(A) Figura 7. Graficación RED-2. Fuente: UCINET V. 6.682

\section{Actores con Capacidad de Transferencia Tecnológica (ACTT) RED 2 - T2}

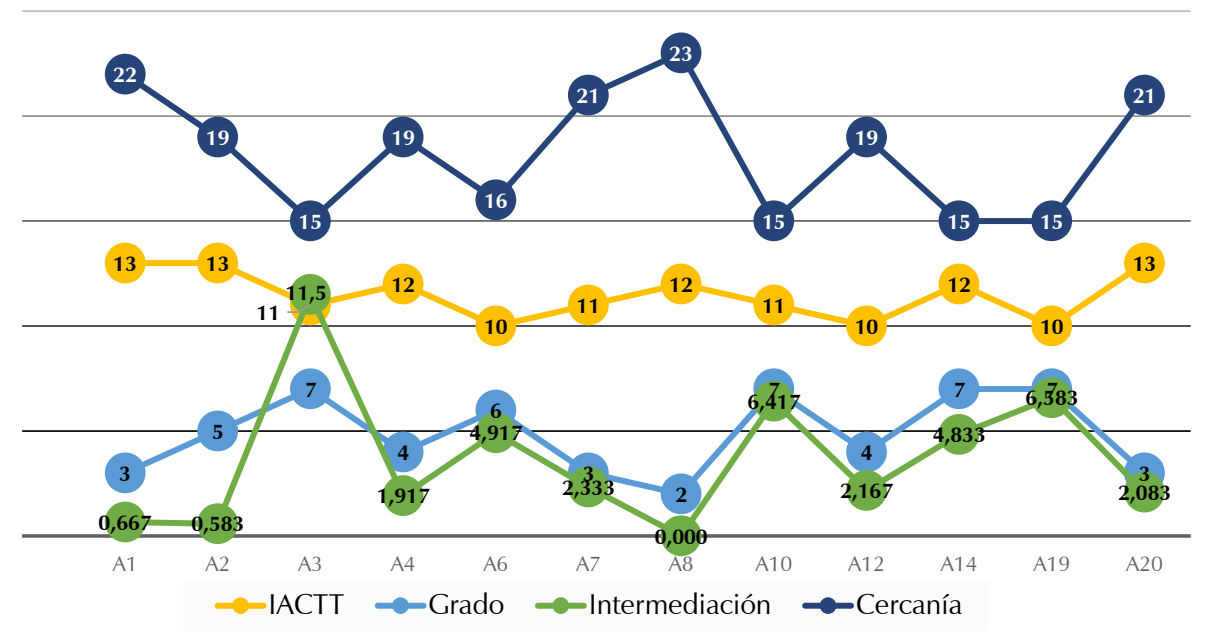

(A) Figura 8. Graficación, RED-2. Fuente: UCINET V. 6.682 
$\rightarrow$ Tabla 7. Resultados RED-3. Fuente: elaboración propia (2019).

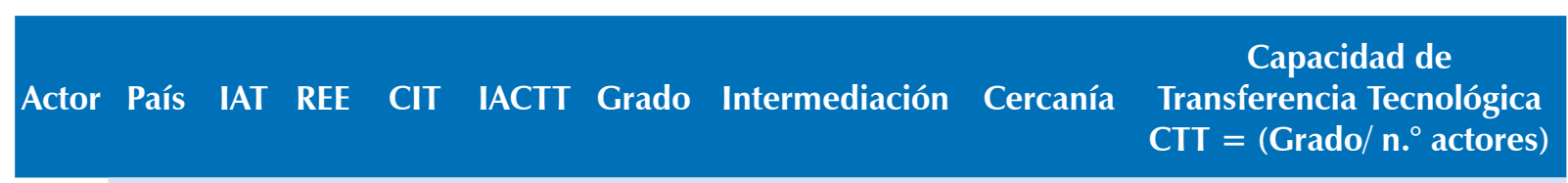

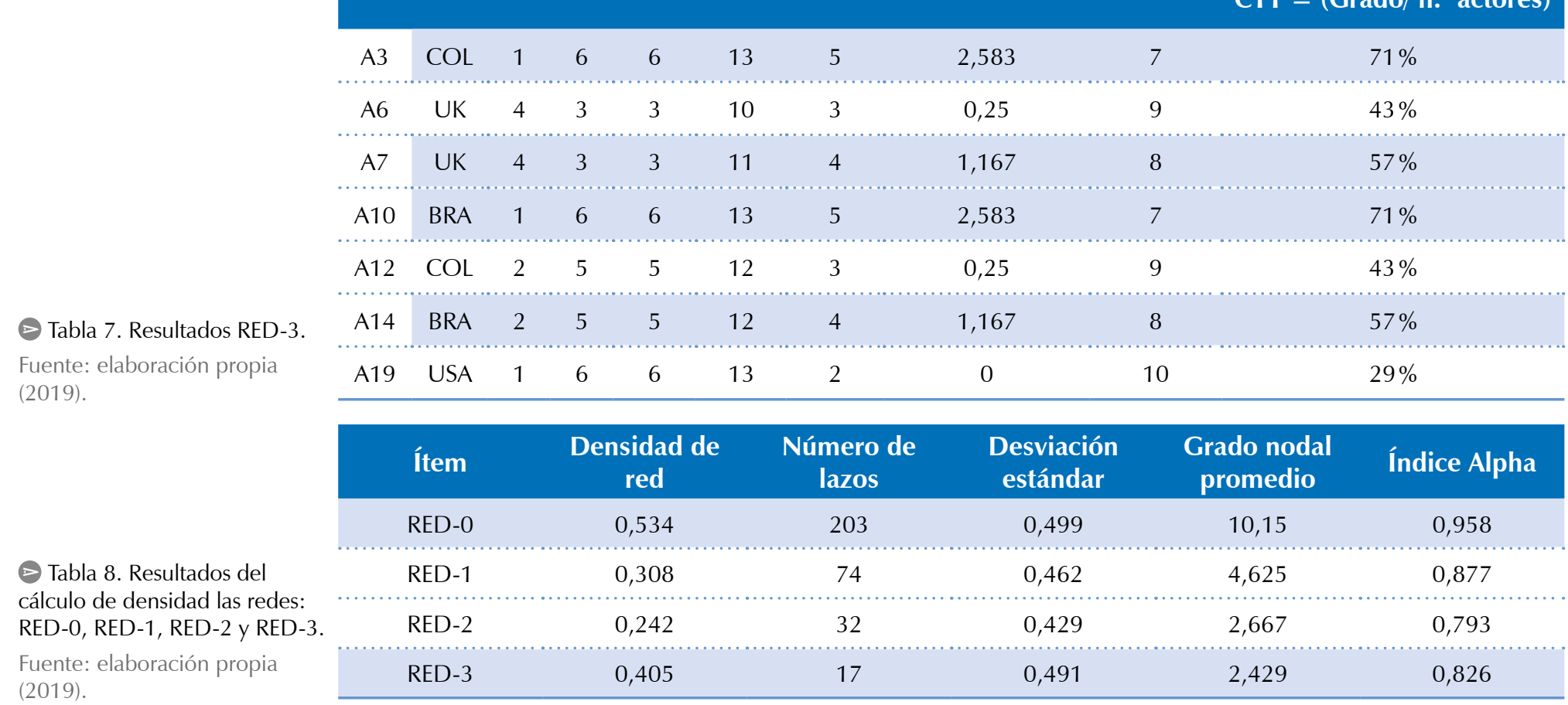

Fuente: elaboración propia (2019).

\section{Resultados de La RED-3}

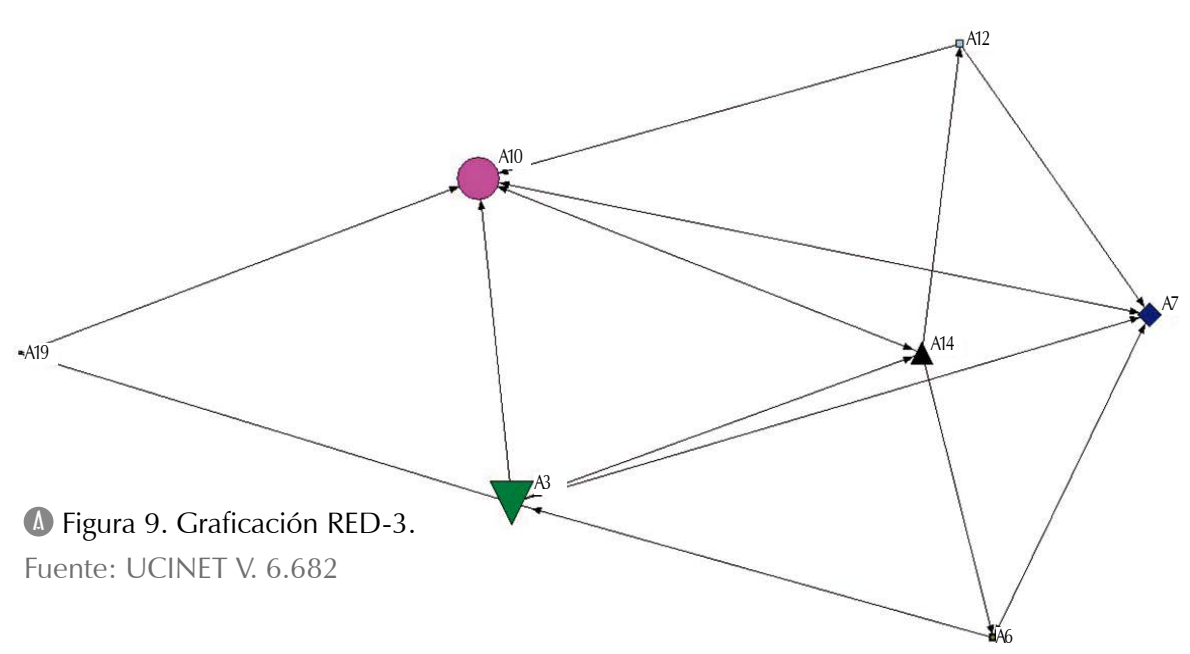

Actores con Capacidad de Transferencia Tecnológica (ACTT) RED 3 - T3

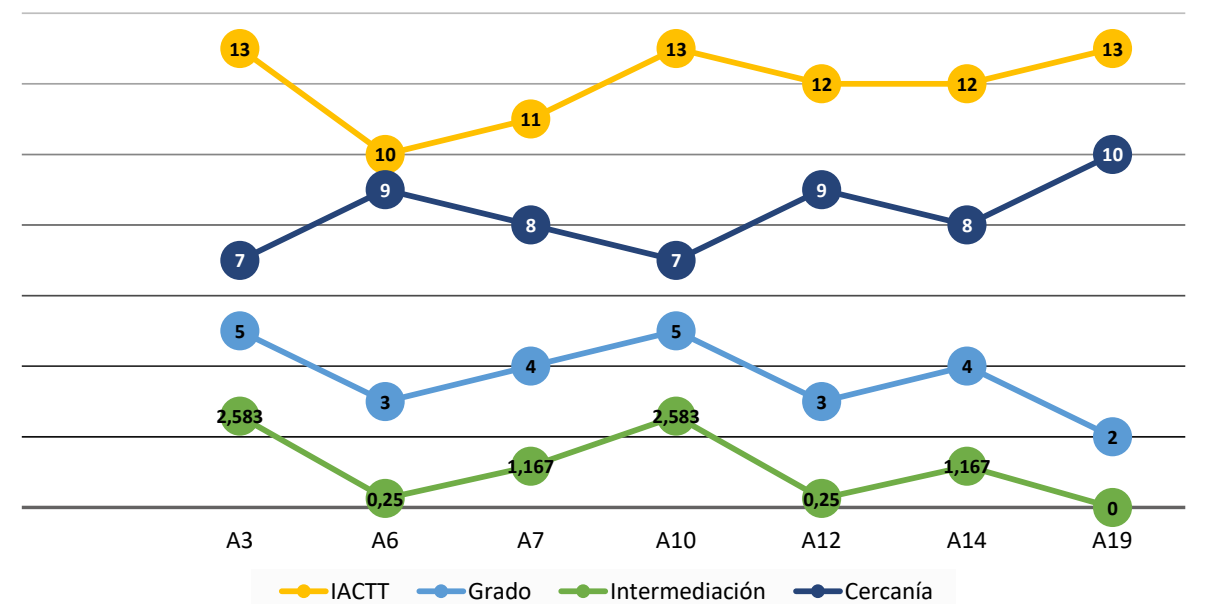

(4) Figura 10. Graficación, RED-3.

Fuente: UCINET V. 6.682
Después de la tercera eliminación de las firmas constructoras que tienen las menores capacidades de transferencia tecnológica, la red inicia con un tiempo T3 y con 7 actores. Se observa que las 4 principales firmas de la red son una europea y 3 latinoamericanas. Se observa que las 4 poseen capacidades similares de transferencia tecnológica. En este caso, las 2 firmas constructoras son A3 y A10, que tienen una capacidad de transferencia tecnológica del $71 \%$ y un IACTT de 13. Ambas firmas logran obtener en el proceso la tecnología más alta en el mercado; además, las 2 firmas tienen una alta intermediación de 2,583. Es decir, las 2 empresas tienen capacidad de transmisión y recepción tecnológica dentro de la red. Por último, en la tabla 7 se pueden observar los 4 primeros actores de la RED-3 y los resultados correspondientes. La figura 9 muestra la graficación de la RED-3 y la figura 10 presenta el comportamiento grafico de los datos de la tabla 7.

Por otro lado, la tabla 8 muestra el cálculo de la densidad de las 4 redes analizadas. La densidad es una medida que calcula el potencial de relación entre los actores de la red; por tanto, con esta medida podemos constatar el potencial de relaciones que se presentan en las 4 redes y su grado promedio. En ella se puede ver que la RED0 inicia con una densidad de 0,534 y termina en la RED-3, con una densidad de 0,405.

Según lo anterior, se presenta una pérdida del $24 \%$ en la densidad de red en todo el proceso de simulación y análisis de redes para el proceso de transferencia de tecnologías sostenibles en las firmas constructoras; además, se identifica una alta reducción del número de oportunidades de relación entre los actores; el número de lazos se reduce de 203 en la RED-0 a 17 en la RED-3 (tabla 8). 
Firmas constructoras de Brasil y Colombia

\begin{tabular}{|c|c|c|c|c|c|c|c|c|c|c|c|c|}
\hline \multirow{2}{*}{ Actor } & \multirow{2}{*}{ País } & \multirow{2}{*}{$\begin{array}{c}\text { RED-0 (T0) } \\
\text { Capacidad } \\
\text { transferencia } \\
\text { tecnológica }\end{array}$} & \multirow{2}{*}{$\begin{array}{c}\text { RED-1 (T1) } \\
\text { Capacidad } \\
\text { transferencia } \\
\text { tecnológica }\end{array}$} & \multirow{2}{*}{$\begin{array}{c}\text { Resultado de la } \\
\text { capaciadad de } \\
\text { transferencia } \\
\text { tecnológica } \\
\text { Pérdida Ganancia }\end{array}$} & \multirow{2}{*}{$\begin{array}{c}\text { RED-1 (T1) } \\
\text { Capacidad } \\
\text { transferencia } \\
\text { tecnológica }\end{array}$} & \multirow{2}{*}{$\begin{array}{c}\text { RED-2 (T2) } \\
\text { Capacidad } \\
\text { transferencia } \\
\text { tecnológica }\end{array}$} & \multicolumn{2}{|c|}{$\begin{array}{l}\text { Resultado de la } \\
\text { capaciadad de } \\
\text { transferencia } \\
\text { tecnológica }\end{array}$} & \multirow{2}{*}{$\begin{array}{c}\text { RED-2 (T2) } \\
\text { Capacidad } \\
\text { transferencia } \\
\text { tecnológica }\end{array}$} & \multirow{2}{*}{$\begin{array}{c}\text { RED-2 (T2) } \\
\text { Capacidad } \\
\text { transferencia } \\
\text { tecnológica }\end{array}$} & \multicolumn{2}{|c|}{$\begin{array}{l}\text { Resultado de la } \\
\text { capaciadad de } \\
\text { transferencia } \\
\text { tecnológica }\end{array}$} \\
\hline & & & & & & & Pérdida & Ganancia & & & Pérdida & Ganancia \\
\hline A10 & BRA & $95 \%$ & $69 \%$ & $26 \%$ & $69 \%$ & $58 \%$ & $10 \%$ & & $58 \%$ & $50 \%$ & $8 \%$ & \\
\hline A3 & $\mathrm{COL}$ & $80 \%$ & $75 \%$ & $5 \%$ & $75 \%$ & $58 \%$ & $17 \%$ & & $58 \%$ & $58 \%$ & $0 \%$ & \\
\hline A14 & BRA & $90 \%$ & $38 \%$ & $53 \%$ & $38 \%$ & $58 \%$ & & $21 \%$ & $58 \%$ & $25 \%$ & $33 \%$ & \\
\hline A12 & $\mathrm{COL}$ & $75 \%$ & $38 \%$ & $38 \%$ & $38 \%$ & $33 \%$ & $4 \%$ & & $33 \%$ & $25 \%$ & $8 \%$ & \\
\hline A2 & $\mathrm{COL}$ & $75 \%$ & $63 \%$ & $13 \%$ & $63 \%$ & $42 \%$ & $21 \%$ & & $42 \%$ & $25 \%$ & $17 \%$ & \\
\hline A4 & $\mathrm{COL}$ & $85 \%$ & $63 \%$ & $23 \%$ & $63 \%$ & $33 \%$ & $29 \%$ & & $33 \%$ & $0 \%$ & $33 \%$ & \\
\hline A8 & BRA & $75 \%$ & $63 \%$ & $13 \%$ & $63 \%$ & $17 \%$ & $46 \%$ & & $17 \%$ & $0 \%$ & $17 \%$ & \\
\hline A18 & BRA & $70 \%$ & $31 \%$ & $39 \%$ & $31 \%$ & $0 \%$ & $31 \%$ & & $0 \%$ & $0 \%$ & $0 \%$ & \\
\hline A17 & $\mathrm{COL}$ & $70 \%$ & $25 \%$ & $45 \%$ & $25 \%$ & $0 \%$ & $25 \%$ & & $0 \%$ & $0 \%$ & $0 \%$ & \\
\hline A13 & BRA & $60 \%$ & $19 \%$ & $41 \%$ & $19 \%$ & $0 \%$ & $19 \%$ & & $0 \%$ & $0 \%$ & $0 \%$ & \\
\hline
\end{tabular}

Firmas constructoras de Reino Unido y Estados Unidos

\begin{tabular}{|c|c|c|c|c|c|c|c|c|c|c|c|c|c|}
\hline \multirow{2}{*}{ Actor } & \multirow{2}{*}{ País } & \multirow{2}{*}{$\begin{array}{c}\text { RED-0 (T0) } \\
\text { Capacidad } \\
\text { transferencia } \\
\text { tecnológica }\end{array}$} & \multirow{2}{*}{$\begin{array}{c}\text { RED-1 (T1) } \\
\text { Capacidad } \\
\text { transferencia } \\
\text { tecnológica }\end{array}$} & \multicolumn{2}{|c|}{$\begin{array}{l}\text { Resultado de la } \\
\text { capaciadad de } \\
\text { transferencia } \\
\text { tecnológica }\end{array}$} & RED-1 (T1) & RED-2 (T2) & \multicolumn{2}{|c|}{$\begin{array}{l}\text { Resultado de la } \\
\text { capaciadad de } \\
\text { transferencia } \\
\text { tecnológica }\end{array}$} & \multirow{2}{*}{$\begin{array}{c}\text { RED-2 (T2) } \\
\text { Capacidad } \\
\text { transferencia } \\
\text { tecnológica }\end{array}$} & \multirow{2}{*}{$\begin{array}{c}\text { RED-3 (T3) } \\
\text { Capacidad } \\
\text { transferencia } \\
\text { tecnológica }\end{array}$} & \multicolumn{2}{|c|}{$\begin{array}{l}\text { Resultado de la } \\
\text { capaciadad de } \\
\text { transferencia } \\
\text { tecnológica }\end{array}$} \\
\hline & & & & Pérdida & Ganancia & $\begin{array}{c}\text { Capacidad } \\
\text { transferencia } \\
\text { tecnológica }\end{array}$ & $\begin{array}{c}\text { Capacidad } \\
\text { transferencia } \\
\text { tecnológica }\end{array}$ & Pérdida & Ganancia & & & Pérdida & Ganancia \\
\hline A6 & UK & $75 \%$ & $44 \%$ & $31 \%$ & & $44 \%$ & $50 \%$ & & $6 \%$ & $50 \%$ & $43 \%$ & $7 \%$ & \\
\hline A19 & USA & $80 \%$ & $44 \%$ & $36 \%$ & & $44 \%$ & $58 \%$ & & $15 \%$ & $58 \%$ & $29 \%$ & $30 \%$ & \\
\hline A7 & UK & $70 \%$ & $44 \%$ & $26 \%$ & & $44 \%$ & $25 \%$ & $19 \%$ & & $25 \%$ & $57 \%$ & & $32 \%$ \\
\hline A1 & UK & $65 \%$ & $75 \%$ & & $10 \%$ & $75 \%$ & $25 \%$ & $50 \%$ & & $25 \%$ & $0 \%$ & $25 \%$ & \\
\hline A20 & USA & $75 \%$ & $50 \%$ & $25 \%$ & & $50 \%$ & $25 \%$ & $25 \%$ & & $25 \%$ & $0 \%$ & $25 \%$ & \\
\hline A11 & USA & $70 \%$ & $25 \%$ & $45 \%$ & & $25 \%$ & $0 \%$ & $25 \%$ & & $0 \%$ & $0 \%$ & $0 \%$ & \\
\hline A5 & UK & $65 \%$ & $0 \%$ & $65 \%$ & & $0 \%$ & $0 \%$ & $0 \%$ & & $0 \%$ & $0 \%$ & $0 \%$ & \\
\hline A9 & UK & $65 \%$ & $0 \%$ & $65 \%$ & & $0 \%$ & $0 \%$ & $0 \%$ & & $0 \%$ & $0 \%$ & $0 \%$ & \\
\hline A15 & USA & $65 \%$ & $0 \%$ & $65 \%$ & & $0 \%$ & $0 \%$ & $0 \%$ & & $0 \%$ & $0 \%$ & $0 \%$ & \\
\hline A16 & USA & $65 \%$ & $0 \%$ & $65 \%$ & & $0 \%$ & $0 \%$ & $0 \%$ & & $0 \%$ & $0 \%$ & $0 \%$ & \\
\hline
\end{tabular}

Por último, en la tabla 9 se presentan los resultados de las pérdidas y las ganancias en la capacidad de transferencia tecnológica de las 20 firmas analizadas a través de redes. De las firmas constructoras de Brasil y Colombia, se destaca el actor A14, de Brasil, que presenta una ganancia del $21 \%$ en su capacidad de transferencia tecnológica en la transición entre la RED-1 y la RED2. Mientras, el actor A6, del Reino Unido, y el A19, de Estados Unidos, muestran una ganancia del $6 \%$ y el $15 \%$, respectivamente, en su capacidad de transferencia tecnológica en la transición entre la RED-1 y la RED-2.

Por otro lado, el actor A7, del Reino Unido, muestra una ganancia del $32 \%$ en su capaci- dad de transferencia tecnológica en la transición entre la RED-2 y la RED-3. Finalmente, el actor A1 exhibe una ganancia del $10 \%$ en su capacidad de transferencia tecnológica.

\section{Conclusiones}

Con el presente estudio se pudieron mostrar la complejidad y la relación de las variables involucradas en el proceso de transferencia tecnológica entre distintas firmas de construcción seleccionadas internacionalmente. La identificación de una brecha de investigación en esta área permitió evaluar la transferencia de tecnologías sostenibles entre empresas constructoras que se dedican a construir vivienda social o vivienda accesible.
A Tabla 9. Resultados del cálculo de pérdidas y ganancias de la capacidad de transferencia tecnológica: RED-0, RED-1, RED-2 y RED-3.

Fuente: elaboración propia (2019). 
Por lo anterior, este ejercicio pudo desarrollar un indicador de medición de la transferencia tecnología sostenible entre firmas constructoras. En primer lugar, se concluye que fortalecer las capacidades de transferencia tecnológica permite ganancias en este sentido a medianos y largos plazos para las firmas constructoras.

Por otra parte, sin embargo, se observa que se presenta una pérdida del $24 \%$ en la densidad de red en todo el proceso. También se observa que las firmas constructoras de Brasil y Colombia muestran ganancias del $21 \%$ en su capacidad de transferencias tecnológica a mediano plazo al entrar en contacto con empresas internacionales más fuertes y con mejores tecnologías.

Así mismo, se observa que algunas firmas pueden presentar pequeños retrocesos tecnológicos mientras desarrollan el proceso de fortalecimiento de su capacidad de transferencia tecnológica.

Este ejercicio evidencia la capacidad de adaptación que presentan las firmas constructoras latinoamericanas frente a la capacidad de transferencia de las firmas constructoras de países industrializados. En este punto, podemos confirmar que la hipótesis propuesta al inicio de este artículo es válida.

\section{Referencias}

Abbasian-Hosseini, S. A., Liu, M., \& Hsiang, S. M. (20 15). Social network analysis for construction specialty trade interference and work plan reliability. Proceedings of IGLC 23 - 23rd Annual Conference of the International Group for Lean Construction: Global Knowledge - Global Solutions, 2015-January (919), 143-152. Recuperado de:: http://iglc.net/Papers/Details/1223

Alarcón, D. M., Alarcón, I. M., \& Alarcón, L. F. (2013). Social network analysis: A diagnostic tool for information flow in the AEC industry. 21st Annual Conference of the International Group for Lean Construction 2013 IGLC 2013, 196-205. Recuperado de: http://iglc.net/Papers/Details/864

Alsema, E. A., Anink, D., Meijer, A., Straub, A., \& Donze, G. (2016). Integration of Energy and Material Performance of Buildings: $I=E+M$. Energy Procedia, 96(October), 517-528. Doi:https://doi.org/10.1016/j.egypro.2016.09.094

Asadi, E., Silva, M. G. Da, Antunes, C. H., Dias, L., \& Glicksman, L. (2014). Multi-objective optimization for building retrofit: A model using genetic algorithm and artificial neural network and an application. Energy and Buildings, 81, 444-456.

Borgatti, S.P., Everett, M.G., \& Johnson, J.C. (2013). Analyzing Social Networks. Sage Publications.

Carlucci, S., Lobaccaro, G., Li, Y., Catto Lucchino, E., \& Ramaci, R. (2016). The effect of spatial and temporal randomness of stochastically generated occupancy schedules on the energy performance of a multiresidential building. Energy and Buildings, 127, 279-300.

https://doi.org/10.1016/j.enbuild.2016.05.023

Castillo, T., Alarcón, L. F., \& Pellicer, E. (2018). Influence of Organizational Characteristics on Construction Project Performance Using Corporate Social Networks. Journal of Management in Engineering, 34(4).

Doi:https://doi.org/10.1061/(ASCE)ME.19435479.0000612

Gelesz, A., \& Reith, A. (2015). Climate-based performance evaluation of double skin facades by building energy modelling in Central Europe. Energy Procedia, 78, 555-560. Doi:https://doi.org/10.1016/j.egypro.2015.11.735

Huang, I. B., Keisler, J., \& Linkov, I. (2011). Multicriteria decision analysis in environmental sciences: Ten years of applications and trends. Science of the Total Environment, 409(19), 3578-3594.

https://doi.org/10.1016/j.scitotenv.2011.06.022
Kim, M. J., Oh, M. W., \& Kim, J. T. (2013). A method for evaluating the performance of green buildings with a focus on user experience. Energy and Buildings, 66, 203-210. https://doi.org/10.1016/j.enbuild.2013.07.049

Kontu, K., Rinne, S., Olkkonen, V., Lahdelma, R., \& Salminen, P. (2015). Multicriteria evaluation of heating choices for a new sustainable residential area. Energy and Buildings, 93(x), 169-179.

https://doi.org/10.1016/j.enbuild.2015.02.003

Liu, Y., Guo, X., \& Hu, F. (2014). Cost-benefit analysis on green building energy efficiency technology application: A case in China. Energy and Buildings, 82, 37-46. https://doi.org/10.1016/j.enbuild.2014.07.008

Lopes, R. A., Chambel, A., Neves, J., Aelenei, D., \& Martins, J. (2016). A Literature Review of Methodologies Used to Assess the Energy Flexibility of Buildings. Energy Procedia, 91, 1053-1058.

Doi:https://doi.org/10.1016/j.egypro.2016.06.274

Ma, H., Zhou, W., Lu, X., Ding, Z., \& Cao, Y. (2016). Application of Low Cost Active and Passive Energy Saving Technologies in an Ultra-low Energy Consumption Building. Energy Procedia, 88, 807-813.

Doi:https://doi.org/10.1016/j.egypro.2016.06.132

Marques, S. B., Bissoli-Dalvi, M., \& Alvarez, C. E. de. (2018). Políticas públicas em prol da sustentabilidade na construção civil em municípios brasileiros. urbe. Revista Brasileira de Gestão Urbana, 10(Suppl. 1), 186-196. Epub July 30, 2018.

Doi:https://dx.doi.org/10.1590/21753369.010.supl1.ao10

McKinsey Global Institute. (2017). Reinventing Construction: A Route to Higher Productivity. McKinsey \& Company, (February), 20. https://www.mckinsey.com/ /media/ McKinsey/Industries/Capital Projects and Infrastructure/Our Insights/Reinventing construction through a productivity revolution/MGI-Reinventing-construction-Aroute-to-higher-productivity-Full-report.pdf

Moschetti, R., \& Brattebø, H. (2016). Sustainable business models for deep energy retrofitting of buildings: state-of-the-art and methodological approach. Energy Procedia, 96(1876), 435-445.

https://doi.org/10.1016/j.egypro.2016.09.174

Niknam, M., \& Karshenas, S. (2015). Sustainable Design of Buildings using Semantic BIM and Semantic Web Services. Procedia Engineering, 118, 909-917. https://doi.org/10.1016/j.proeng.2015.08.530

Panchal, S., Dincer, I., \& Agelin-Chaab, M. (2016). Analysis and evaluation of a new renewable energy based integrated system for residential applications. Energy and Buildings, 128, 900-910.

https://doi.org/10.1016/j.enbuild.2016.07.038

Park, H., \& Han, S. H. (2012). Impact of interfirm collaboration networks in international construction projects: A longitudinal study. In Construction Research Congress 2012: Construction Challenges in a Flat World (pp. 1460-1470). Construction Management and Information Laboratory, Dept. of Civil and Environmental Engineering, Yonsei University, Seoul, South Korea.

Doi:https://doi.org/10.1061/9780784412329.147

Pisello, A. L., Castaldo, V. L., Taylor, J. E., \& Cotana, F. (2016). The impact of natural ventilation on building energy requirement at interbuilding scale. Energy and Buildings, 127, 870-883.

Doi:https://doi.org/10.1016/j.enbuild.2016.06.023

Salcido, J. C., Abdul, A., \& Issa, R. R. A. (2016). From simulation to monitoring: Evaluating the potential of mixed-mode ventilation (MMV) systems for integrating natural ventilation in office buildings through a comprehensive literature review. Energy \& Buildings, 127, 1008-1018.

Doi:https://doi.org/10.1016/j.enbuild.2016.06.054

Sartori, I., Napolitano, A., \& Voss, K. (2012). Net zero energy buildings: A consistent definition framework. Energy and Buildings, 48, 220-232.

https://doi.org/10.1016/j.enbuild.2012.01.032

Zabalza Bribián, I., Valero Capilla, A., \& Aranda Usón, A. (2011). Life cycle assessment of building materials: Comparative analysis of energy and environmental impacts and evaluation of the eco-efficiency improvement potential. Building and Environment, 46(5), 1133-1140.

https://doi.org/10.1016/j.buildenv.2010.12.002

Zucker, G., Judex, F., Blöchle, M., Köstl, M., Widl, E., Hauer, S., ... Zeilinger, J. (2016). A new method for optimizing operation of large neighborhoods of buildings using thermal simulation. Energy and Buildings, 125, 153-160.

https://doi.org/10.1016/j.enbuild.2016.04.081 


\section{Vol.}
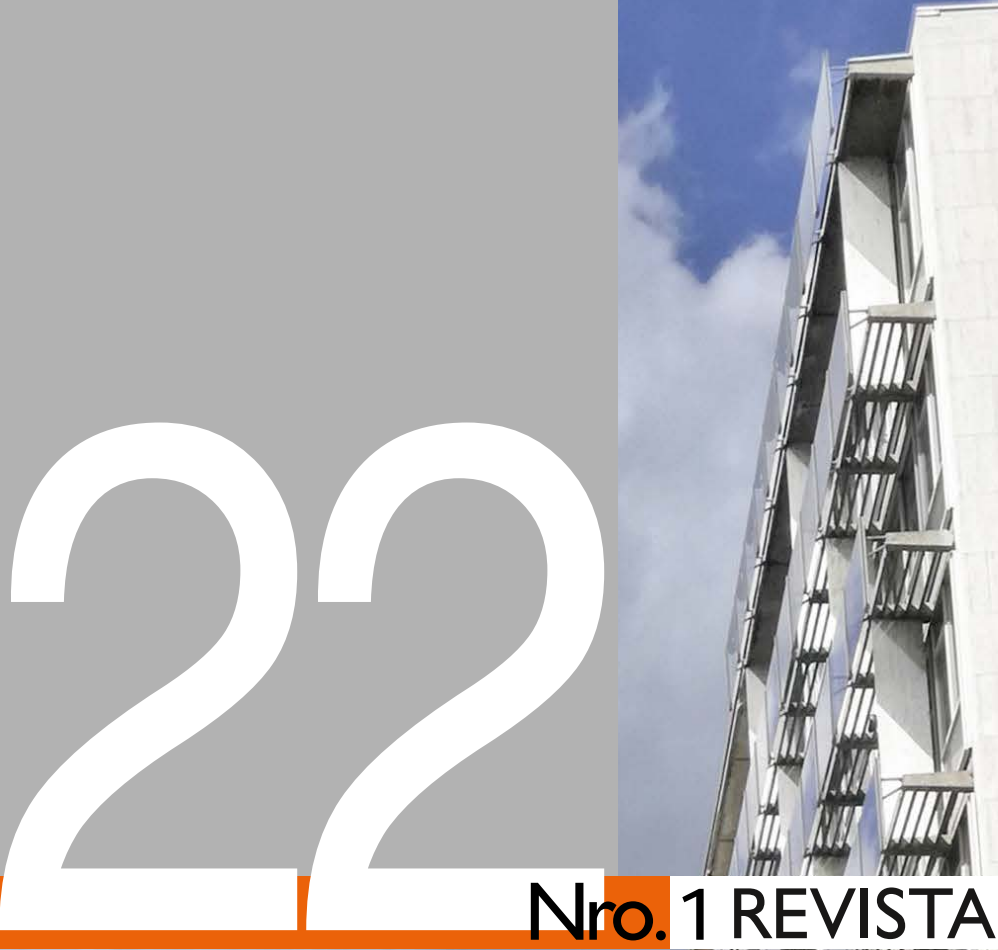

ISSN: 1657-0308 (Impresa)

Nro. 1 REVISTA DE ARQUITECTURA

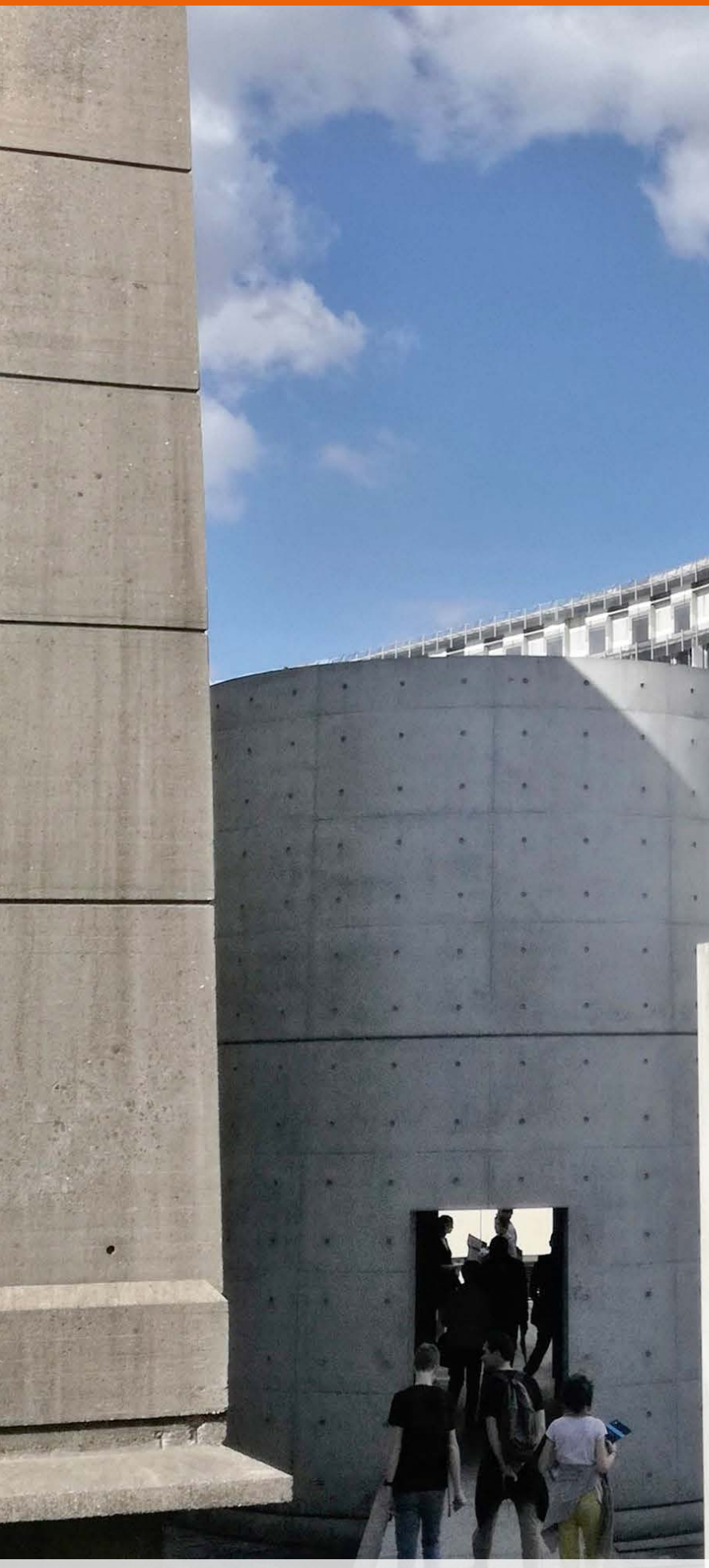

$\overline{9}$
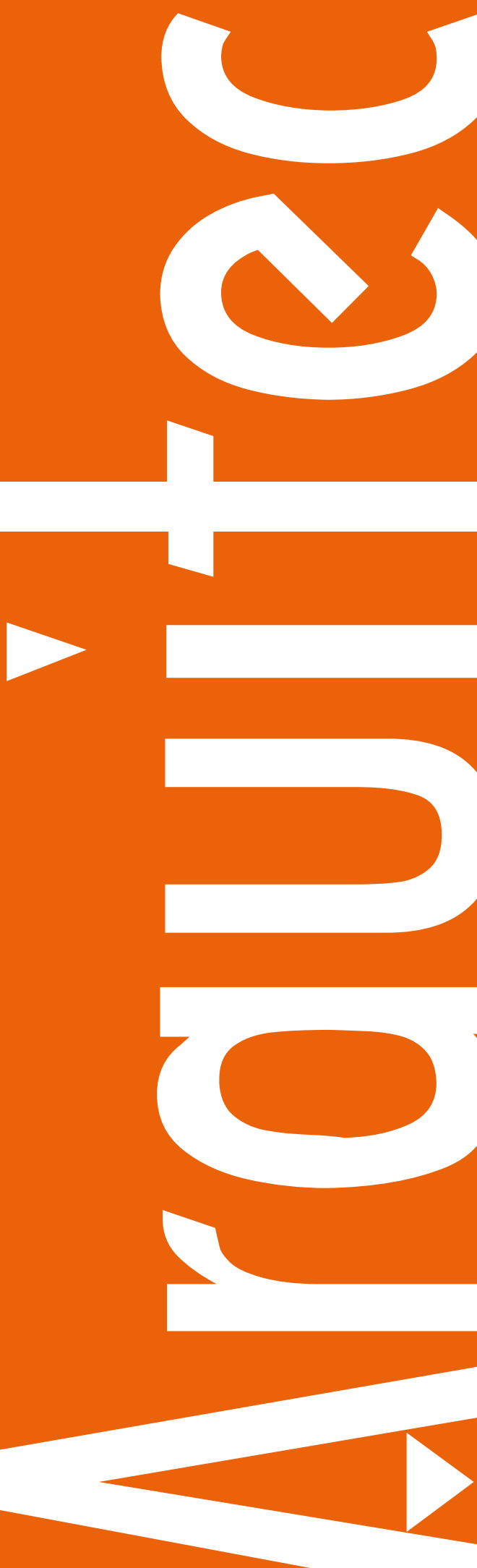


\section{Enfoque y alcance}

La Revista de Arquitectura (Bogotá) ( (ISSN 1657-0308 Impresa y E-ISSN 2357-626X en línea) es una publicación científica seriada de acceso abierto, arbitrada mediante revisión por pares (doble ciego) e indexada, en donde se publican resultados de investigación originales e inéditos.

Está dirigida a la comunidad académica y profesional de las áreas afines a la disciplina. Es editada por la Facultad de Diseño y el Centro de Investigaciones (CIFAR) de la Universidad Católica de Colombia en Bogotá (Colombia).

La principal área científica a la que se adscribe la Revisto de Arquitectura (Bogotá) según la OCDE es:

Gran área: 6. Humanidades

Área: 6.D. Arte

Disciplina: 6D07. Arquitectura y Urbanismo

También se publican artículos de las disciplinas como 2A02, Ingeniería arquitectónica; 5C03, Estudios urbanos (planificación y desarrollo); 6D07, Diseño.

Los objetivos de la Revista de Arquitectura (Bogotá) son:

- Promover la divulgación y difusión del conocimiento generado a nivel local, nacional e internacional

- Conformar un espacio para la construcción de comunidades académicas y la discusión en torno a las secciones definidas.

- Fomentar la diversidad institucional y geográfica de los autores que participan en la publicación.

- Potenciar la discusión de experiencias e intercambios científicos entre investigadores y profesionales.

- Contribuir a la visión integral de la arquitectura, por medio de la concurrencia y articulación de las secciones mediante la publicación de artículos de calidad.

- Publicar artículos originales e inéditos que han pasado por revisión de pares, para asegurar que se cumplen las normas éticas, de calidad, validez científica, editorial e investigativa.

- Fomentar la divulgación de las investigaciones y actividades desarrolladas en la Universidad Católica de Colombia.
Palabras clave de la Revista de Arquitectura (Bogotá): arquitectura, diseño, educación arquitectónica, proyecto y construcción, urbanismo.

Idiomas de publicación: español, inglés, portugués y francés. Título abreviado: Rev. Arquit.

\section{Titulo corto: RevArq}

\section{Políticas de sección}

La revista se estructura en tres secciones correspondientes a las líneas de investigación activas y aprobadas por la institución, y dos complementarias, que presentan dinámicas propias de la Facultad de Diseño y las publicaciones relacionadas con la disciplina.

Cultura y espacio urbano. En esta sección se publican los artículos que se refieren a fenómenos sociales en relación con el espacio urbano, atendiendo aspectos de la historia, el patrimonio cultural y físico, y la estructura formal de las ciudades y el territorio.

Proyecto arquitectónico y urbano. En esta sección se presentan artículos sobre el concepto de proyecto, entendido como elemento que define y orienta las condiciones proyectuales que devienen en los hechos arquitectónicos o urbanos, y la forma como estos se convierten en un proceso de investigación y nuevo de conocimiento. También se presentan proyectos que sean resultados de investigación, los cuales se validan por medio de la ejecución y transformación en obra construida del proceso investigativo. También se contempla la publicación de investigaciones relacionadas con la pedagogía y didáctica de la arquitectura, el urbanismo y el diseño.

Tecnología, medioambiente y sostenibilidad. En esta sección se presentan artículos acerca de sistemas estructurales, materiales y procesos constructivos, medioambiente y gestión, relacionados con los entornos social-cultural, ecológico y económico.

Desde la Facultad. En esta sección se publican artículos generados en la Facultad de Diseño, relacionados con las actividades de docencia, extensión, formación en investigación o internacionalización, las cuales son reflejo de la dinámica y de las actividades realizadas por docentes, estudiantes y egresados; esta sección no puede superar el $20 \%$ del contenido.

Textos. En esta sección se publican reseñas, traducciones y memorias de eventos relacionados con las publicaciones en Arquitectura y Urbanismo.
A Frecuencia de publicación

Desde 1999 y hasta el 2015, la Revista de Arquitectura (Bogotá) publicó un volumen al año, a partir del 2016 se publicarán dos números por año en periodo anticipado, enero-junio y julio-diciembre, pero también maneja la publicación anticipada en línea de los artículos aceptados (versión Post-print del autor).

La Revista de Arquitectura (Bogotá) se divulga mediante versiones digitales (PDF, HTML, EPUB, XML) e impresascon un tiraje de 700 ejemplares, los tiempos de producción de estas versiones dependerán de los cronogramas establecidos por la editorial.

Los tiempos de recepción-revisión-aceptación pueden tardar entre seis y doce meses dependiendo del flujo editorial de cada sección y del proceso de revisión y edición adelantado.

Con el usuario y contraseña asignados, los autores pueden ingresar a la plataforma de gestión editorial y verificar el estado de revisión, edición o publicación del artículo.

\section{A Canje}

La Revista de Arquitectura (Bogotá) está interesada en establecer canje con publicaciones académicas, profesionales o científicas del área de Arquitectura y Urbanismo, como medio de reconocimiento y discusión de la producción científica en el campo de acción de la publicación.

\section{Mecanismo}

Para establecer canje por favor descargar, diligenciar y enviar el formato: RevArq FP20 Canjes
Universidad Católica de Colombia (2020, enero-junio). Revista de Arquitectura (Bogotá), 22(I) I-188. Doi: 10.14718

ISSN: 1657-0308 E-ISSN: 2357-626X

Especificaciones:

Formato: $34 \times 24 \mathrm{~cm}$

Papel: Mate $115 \mathrm{~g}$

Tintas: Negro y policromía
A Contacto

Dirección postal:

Avenida Caracas No. 46-72.

Universidad Católica de Colombia

Bogotá D.C.(Colombia)

Código postal: 111311

Facultad de Diseño Centro de Investigaciones (CIFAR). Sede El Claustro. Bloque " $\mathrm{L}$ ", 4 piso Diag. 46A No. $15 \mathrm{~b}-10$ Editor, Arq. César Eligio-Triana

Teléfonos:

+57 (1) $3277300-3277333$

Ext. 3109; 3112 o 5146

Fax: +57 (1) 2858895
Correo electrónico: revistadearquitectura@ucatolica.edu.co cifar@ucatolica.edu.co

Página WEB: www.ucatolica.edu.co vínculo Revistas científicas

http://publicaciones.ucatolica.edu.co revistas-cientificas http://editorial.ucatolica.edu.co/ojsucatolica/revistas ucatolica/index.php/RevArq 
